\title{
A PRIMAL-DUAL ACTIVE SET ALGORITHM FOR THREE-DIMENSIONAL CONTACT PROBLEMS WITH COULOMB FRICTION*
}

\author{
S. HÜEBER, G. STADLER AND B.I. WOHLMUTH
}

\begin{abstract}
In this paper, efficient algorithms for contact problems with Tresca and Coulomb friction in three dimensions are presented and analyzed. The numerical approximation is based on mortar methods for nonconforming meshes with dual Lagrange multipliers. Using a nonsmooth complementarity function for the 3D friction conditions, a primal-dual active set algorithm is derived. The method determines active contact and friction nodes and, at the same time, resolves the additional nonlinearity originating from sliding nodes. No regularization and no penalization is applied, and local superlinear convergence can be observed. In combination with a multigrid method, it defines a robust and fast strategy for contact problems with Tresca or Coulomb friction. The efficiency and flexibility of the method is illustrated by several numerical examples.
\end{abstract}

KeYwords: 3D Coulomb friction, contact problems, dual Lagrange multipliers, inexact primal-dual active set strategy, semismooth Newton methods, nonlinear multigrid method.

AMS Subject Classification (2000): 65K10, 65C20, 65N30, 65N55, 74M15 .

\section{Introduction}

Solving contact problems with friction in $3 \mathrm{D}$ is a challenging task in mechanics and of crucial importance in various applications. The main difficulty lies in the conditions for contact and friction, which are inherently nonlinear and complicate the theoretical analysis and the design of an efficient numerical solution algorithm. For a general introduction on contact problems with and without friction, we refer to [KO88, HHNL88, Wri02, Lau02, Wil03, EJK05].

Very often, laws named after Tresca and Coulomb are used to model friction. It is well known that contact with Tresca friction leads to a classical variational inequality. For Coulomb friction, the friction bound depends on the solution, and this more realistic law results in a quasi-variational inequality.

${ }^{*}$ This work was supported in part by the Deutsche Forschungsgemeinschaft, SFB 404, B8, and SPP 1146.

Received September 29, 2006. 
A widely used approach for contact problems with Coulomb friction is to apply a sequence of Tresca friction problems together with a fixed point iteration (see, e.g., [HKD04,HDK02,LPR91,KS05,FK06,NJH80]). Thus, a crucial component for the solver is a fast and robust algorithm for Tresca frictional contact problems. While in two dimensions, Tresca friction corresponds to linear pointwise inequality constraints for the boundary stresses, the situation in $3 \mathrm{D}$ is more involved due to the quadratic inequality constraint, one has to deal with.

Contributions to theoretically sound numerical algorithms for friction in $3 \mathrm{D}$ are quite rare. We refer to the recent papers [HKD04, $\left.\mathrm{DHK}^{+} 05\right]$, where discrete 3D-Coulomb friction problems are approximated using FETI domain decomposition and algorithms for quadratic programming with simple inequality constraints. Since quadratic rather than linear constraints arise in 3D, they are approximated as intersections of rotated squares in order to make the application of optimization algorithms for simple bounds possible. Improvements of this approximation technique are proposed in [Kuč06a, Kuč06b]. A different idea is followed in [KK01, FK06], where monotone multigrid methods are used to construct a globally convergent solver for discrete Tresca and Coulomb frictional contact problems. The implementation of these methods relies on a multilevel hierarchy of spaces and requires the use of modified coarse grid basis functions and suitable coarse grid constraints.

Already in an early paper [AC91], Newton-type methods for contact problems with friction are used. Similar methods are also studied in the more recent contributions [CKPS98, CP99], where the performance of generalized Newton-type methods for frictional contact problems is shown to be superior to interior point methods. The methods presented in [AC91, CKPS98, CP99] rely on reformulations of contact and friction conditions using nonsmooth equations and on generalized differentiability concepts and, thus, are related to the present paper. However, we use different reformulations of the complementarity conditions, study and exploit the structures arising in Newtontype steps and relate them to (inexact) primal-dual active set strategies. In addition, we apply our algorithm to two-body contact problems which are discretized in terms of mortar techniques and introduce stabilized inexact Newton versions.

Recently, the application of mortar methods as a discretization for the continuous problem has gained a considerable amount of interest (see, e.g., 
[BB00, HL02, HW05, FW05, FW06, Lau02, PL04]). By using a dual basis function for the Lagrange multiplier, the weak form of the constraints can be written as independent constraints for each node on the slave side resulting in a quasi-variational inequality. Using a suitable local basis transformation, only the coefficients on the slave side are affected by the non-penetration condition and the friction constraints. Thus, the algebraic structure of a twobody contact problem is exactly the same as for a one-body contact problem. For details we refer to [WK03, HW05]. In the mixed formulation which is motivated by the mortar approach, the Lagrange multipliers are treated as independent variables. This motivates the application of algorithms, such as the primal-dual active set strategy which use both types of variables. We refer to [HIK03] where the relation between this algorithm and a semismooth Newton method applied to a complementarity function is studied. These methods have been extended and applied successfully to contact problems without friction [HW05, HKK04] and in 2D to friction [HMW06,Sta04,KS05]. However, the application to friction problems in 3D is not at all straightforward. In this paper, we derive a primal-dual active set method by applying a semismooth Newton method to a nonlinear complementarity function that expresses the 3D Tresca friction conditions. In each step of the algorithm, we solve a linear problem with Robin boundary conditions on a suitable subset of the friction boundary and with Dirichlet conditions on the complement. This linear system can be solved using any efficient iterative or fast direct solver. Here, we apply a multigrid method as iterative solver. By solving these systems only approximately, we obtain an inexact primal-dual active set strategy. The resulting algorithm can be regarded as a nonlinear multigrid method; it also applies to problems with Coulomb friction and yields a reliable and fast convergence. Finally, we present and study a full Newton approach for the Coulomb friction problem that avoids fixed point ideas. Our implementation is based on the finite element toolbox $\mathrm{UG}$, see $\left[\mathrm{BBJ}^{+} 97\right.$, and the direct solver PARDISO, see [SGF00], is used for the full Newton approach.

The outline of this paper is as follows: The two-body Coulomb frictional contact problem is stated in Section 2, and the discretization based on mortar methods is briefly discussed. In Section 3, we develop and analyze our algorithm for 3D friction problems. For simplicity of presentation, we restrict ourselves to the case of a one-body contact problem with Tresca friction. In Section 4, we study the performance of the algorithm for a test problem with 
Tresca friction. Using fixed point ideas, this approach is extended to contact problems with Coulomb friction in Section 5. In Section 6, we apply our algorithms to two-body contact problems with Coulomb friction in 3D using a fixed point method. Finally in Section 7, we present a full Newton approach for Coulomb friction in 3D and compare numerically its performance with the discussed fixed point-based methods.

\section{Contact problem with Coulomb friction in 3D}

In this section, we formulate the contact problem with Coulomb friction in linear elasticity. In addition, we briefly describe our nonconforming discretization based on mortar methods and the basis transformation which transforms the system for two bodies into a system having exactly the same structure as a one-body contact problem.

2.1. Problem statement. Let $\Omega^{l} \subset \mathbb{R}^{3}, l \in\{m, s\}$, denote two elastic bodies. The superscript "l" will refer to $s$, for the slave, or to $m$, for the master body, as is common in the mortar setting. We assume that the boundaries $\partial \Omega^{l}$ are divided into three disjoint measurable parts $\Gamma_{D}^{l}, \Gamma_{N}^{l}$ and $\Gamma_{C}^{l}$ with meas $\left(\Gamma_{D}^{l}\right) \neq 0$. We impose homogeneous Dirichlet conditions on $\Gamma_{D}^{l}$ and Neumann data, i.e., a surface traction $\boldsymbol{p}^{l} \in\left(L^{2}\left(\Gamma_{N}^{l}\right)\right)^{3}$ on $\Gamma_{N}^{l}$. Moreover, we denote by $\boldsymbol{f}^{l} \in\left(L^{2}\left(\Omega^{l}\right)\right)^{3}$ the volume forces acting on $\Omega^{l}$. We use the constitutive law for linear elasticity, namely

$$
\boldsymbol{\sigma}^{l}:=\lambda^{l} \operatorname{tr}\left(\varepsilon^{l}\right) \operatorname{Id}+2 \mu^{l} \varepsilon^{l} \quad \text { in } \quad \Omega^{l},
$$

where $\boldsymbol{\sigma}^{l}$ denotes the stress field and $\varepsilon^{l}:=1 / 2\left(\nabla \boldsymbol{u}^{l}+\nabla \boldsymbol{u}^{l^{\top}}\right)$ the linearized strain tensor that both depend on the displacement $\boldsymbol{u}^{l}$. The Lamé constants $\lambda^{l}, \mu^{l}>0$ are given by $\mu^{l}:=E^{l} /\left(2\left(1+\nu^{l}\right)\right)$ and $\lambda^{l}:=\left(E^{l} \nu^{l}\right) /\left(1-\left(\nu^{l}\right)^{2}\right)$ with Young's modulus $E^{l}>0$ and Poisson ratio $\nu^{l} \in(0,0.5)$. Moreover, "tr" denotes the matrix trace operator and "Id" the identity matrix. Then, the displacement $\boldsymbol{u}^{l}$ satisfies the following equations

$$
\begin{aligned}
\operatorname{Div} \boldsymbol{\sigma}^{l}+\boldsymbol{f}^{l} & =0 \text { in } \Omega^{l}, \\
\boldsymbol{u}^{l} & =0 \text { on } \Gamma_{D}^{l}, \\
\boldsymbol{\sigma}^{l} \boldsymbol{n}^{l}-\boldsymbol{p}^{l} & =0 \text { on } \Gamma_{N}^{l},
\end{aligned}
$$

where $\boldsymbol{n}^{l}$ denotes the unit normal outward vector on the boundary $\partial \Omega^{l}$. To state the contact and friction conditions, we introduce for each point of $\Gamma_{C}^{s}$ the vectors $\boldsymbol{\tau}_{1}, \boldsymbol{\tau}_{2}$ that span the tangential plane and use $\boldsymbol{n}:=\boldsymbol{n}^{s}$. We 
assume that $\left\{\boldsymbol{n}, \boldsymbol{\tau}_{1}, \boldsymbol{\tau}_{2}\right\}$ is an orthonormal basis in $\mathbb{R}^{3}$ for each point of $\Gamma_{C}^{s}$. In order to formulate the non-penetration condition of the two bodies, we use a predefined relation between the points of the possible contact zones $\Gamma_{C}^{l}$. This relation is realized by a smooth mapping $R: \Gamma_{C}^{s} \rightarrow \Gamma_{C}^{m}$ satisfying $R\left(\Gamma_{C}^{s}\right) \subset \Gamma_{C}^{m}$. We assume that the mapping $R$ is well defined and maps any $x \in \Gamma_{C}^{s}$ to the intersection of the normal on $\Gamma_{C}^{s}$ at $x$ with $\Gamma_{C}^{m}$. Then the contact conditions on $\Gamma_{C}^{s}$ are given by

$$
[u]_{n}-d \leq 0, \quad \sigma_{n} \leq 0, \quad\left([u]_{n}-d\right) \sigma_{n}=0 \quad \text { on } \Gamma_{C}^{s},
$$

where $d \geq 0$ denotes the gap between the two elastic bodies, $\sigma_{n}:=\boldsymbol{n}^{\top} \boldsymbol{\sigma}^{s} \boldsymbol{n}$ the normal component of the boundary stress, $[\boldsymbol{u}]:=\left(\boldsymbol{u}^{s}(x)-\boldsymbol{u}^{m}(R(x))\right)$ the jump and $[u]_{n}:=[\boldsymbol{u}] \boldsymbol{n}$ the jump in normal direction. For the boundary stress in normal direction on the possible contact part, we have to satisfy the condition

$$
\sigma_{n}=\boldsymbol{n}^{\top} \boldsymbol{\sigma}^{s}(x) \boldsymbol{n}=\boldsymbol{n}^{\top} \boldsymbol{\sigma}^{m}(R(x)) \boldsymbol{n} \quad \text { on } \quad \Gamma_{C}^{s} .
$$

Additionally, we have to ensure $\boldsymbol{\tau}_{k}^{\top} \boldsymbol{\sigma}^{s}(x) \boldsymbol{\tau}_{k}=\boldsymbol{\tau}_{k}^{\top} \boldsymbol{\sigma}^{m}(R(x)) \boldsymbol{\tau}_{k}, k=1,2$. Finally, the Coulomb friction law states that

$$
\left\{\begin{array}{l}
\left\|\boldsymbol{\sigma}_{\tau}\right\| \leq \mathfrak{F}\left|\sigma_{n}\right|, \\
\left\|\boldsymbol{\sigma}_{\tau}\right\|<\mathfrak{F}\left|\sigma_{n}\right| \Rightarrow[\boldsymbol{u}]_{\tau}=0, \\
\left\|\boldsymbol{\sigma}_{\tau}\right\|=\mathfrak{F}\left|\sigma_{n}\right| \Rightarrow \exists \beta \geq 0: \boldsymbol{\sigma}_{\tau}=-\beta[\boldsymbol{u}]_{\tau},
\end{array} \quad \text { on } \Gamma_{C}^{s} .\right.
$$

Above, $\|\cdot\|$ denotes the Euclidean norm in $\mathbb{R}^{3}, \boldsymbol{\sigma}_{\tau}:=\boldsymbol{\sigma}^{s} \boldsymbol{n}-\sigma_{n} \boldsymbol{n}$ the tangential component of the boundary stress and $[\boldsymbol{u}]_{\tau}$ the relative tangential displacement given by $[\boldsymbol{u}]_{\tau}:=[\boldsymbol{u}]-[\boldsymbol{u}]_{n} \boldsymbol{n}$. Furthermore, $\mathfrak{F}: \Gamma_{C}^{s} \rightarrow \mathbb{R}, \mathfrak{F} \geq 0$, is the friction coefficient.

2.2. Mortar discretization. By using dual Lagrange multipliers, we can apply locally a suitable basis transformation for the finite element basis. The shape functions on the master side are modified by adding a linear combination of nodal shape functions on the slave side. In the new basis the shape functions on the master side satisfy a weak continuity condition, i.e., the jump of these basis functions tested with a Lagrange multiplier being defined on the slave side is zero. Thus, the coefficients in this new basis on the slave side of the contact zone describe the relative displacement between the contact interfaces. In this basis, the two-body contact problem has the same structure as in the one body case, since all constraints at the contact zone are restricted to the degrees of freedom on the slave side. For details, 
see [WK03, HW05]. Motivated by these considerations, in the sequel we will write $\boldsymbol{u}_{h}$ for the coefficient vector with respect to the new constrained basis. Therefore, the following discussion holds for both, the one-body and the twobody case. We denote the multiplier corresponding to the discretization of $-\boldsymbol{\sigma}^{s} \boldsymbol{n}$ on the slave side by $\boldsymbol{\lambda}_{h}$. In the new basis, (2.1) is satisfied in the discrete form

$$
A_{h} \boldsymbol{u}_{h}+B_{h} \boldsymbol{\lambda}_{h}=\boldsymbol{f}_{h}
$$

with $B_{h}$ of the form $(0, D)^{\top}$ where due to the use of dual Lagrange multipliers the matrix $D$ is diagonal. Let us denote by $\mathcal{S}$ the set of all nodes of the finite element mesh belonging to $\Gamma_{C}^{s}$ and by $\mathcal{N}$ the set containing all other nodes. Then for each $p \in \mathcal{S}$, the entry of the diagonal matrix $D$ is given by

$$
D[p, p]=I d_{3} \int_{\Gamma_{C}} \phi_{p} \psi_{p} d s=I d_{3} \int_{\Gamma_{C}} \phi_{p} d s=: D_{p} I d_{3},
$$

where $I d_{3}$ denotes the identity matrix in $\mathbb{R}^{3 \times 3}$, and $\phi_{p}$ and $\psi_{p}$ the primal and dual basis function associated with the node $p$, respectively. If the displacements $\boldsymbol{u}_{h}$ are known, the Lagrange multiplier can be computed directly by using (2.5)

$$
\boldsymbol{\lambda}_{h}=D^{-1}\left(\boldsymbol{f}_{h}-A_{h} \boldsymbol{u}_{h}\right)_{\mathcal{S}}
$$

where the subscript $\mathcal{S}$ on the right hand side indicates that we use only the entries of the vector corresponding to the nodes $p \in \mathcal{S}$.

We next introduce the scaled normal and tangential components of the multiplier $\boldsymbol{\lambda}_{h}$ and the normal and tangential components of the relative deformation $\boldsymbol{u}_{h}$. For a node $p \in \mathcal{S}$, let

$$
\begin{gathered}
\boldsymbol{\lambda}_{\tau, p, s}:=\left(\begin{array}{l}
\boldsymbol{\lambda}_{p}^{\top} D_{p} \boldsymbol{\tau}_{1 p} \\
\boldsymbol{\lambda}_{p}^{\top} D_{p} \boldsymbol{\tau}_{2 p}
\end{array}\right) \in \mathbb{R}^{2} \text { and } \lambda_{n, p, s}:=\boldsymbol{\lambda}_{p}^{\top} D_{p} \boldsymbol{n}_{p} \in \mathbb{R}, \\
\boldsymbol{u}_{\tau, p}:=\left(\begin{array}{c}
\boldsymbol{u}_{p}^{\top} \boldsymbol{\tau}_{1 p} \\
\boldsymbol{u}_{p}^{\top} \boldsymbol{\tau}_{2 p}
\end{array}\right) \in \mathbb{R}^{2} \text { and } u_{n, p}:=\boldsymbol{u}_{p}^{\top} \boldsymbol{n}_{p} \in \mathbb{R} .
\end{gathered}
$$

The discretized (and scaled) gap at the node $p \in \mathcal{S}$ is defined as

$$
d_{p}:=\frac{1}{D_{p}} \int_{\Gamma_{C}^{s}} d \psi_{p} d s .
$$

Then, the discrete conditions for normal contact are given by

$$
u_{n, p} \leq d_{p}, \quad \lambda_{n, p, s} \geq 0, \quad \lambda_{n, p, s}\left(u_{n, p}-d_{p}\right)=0
$$


for each $p \in \mathcal{S}$, see also (2.2). We note that we use different scaling factors for the primal and dual variables and that $D_{p}$ is proportional to the local meshsize. This is motivated by the fact that the $H^{-1 / 2}$-norm for the Lagrange multiplier and the $H^{1 / 2}$-norm for the displacements have the same error reduction. We remark that the proposed scaling factors yield better numerical convergence rates for the inexact version of the algorithms. The discrete Coulomb friction conditions are given by

$$
\left\{\begin{array}{l}
\left\|\boldsymbol{\lambda}_{\tau, p, s}\right\| \leq \mathfrak{F}\left|\lambda_{n, p, s}\right|, \\
\left\|\boldsymbol{\lambda}_{\tau, p, s}\right\|<\mathfrak{F}\left|\lambda_{n, p, s}\right| \Rightarrow \boldsymbol{u}_{\tau, p}=0, \\
\left\|\boldsymbol{\lambda}_{\tau, p, s}\right\|=\mathfrak{F}\left|\lambda_{n, p, s}\right| \Rightarrow \exists \beta \geq 0: \boldsymbol{\lambda}_{\tau, p, s}=\beta \boldsymbol{u}_{\tau, p},
\end{array} \quad \text { for all } p \in \mathcal{S} .\right.
$$

Here for simplicity, $\mathfrak{F}$ is assumed to be constant and independent of the solution. Differently from the Coulomb friction conditions (2.4), where the friction bound is $\mathfrak{F}\left|\sigma_{n}\right|$, for Tresca friction this bound is a function $g \in H^{-1 / 2}\left(\Gamma_{C}^{s}\right)$, $g \geq 0$, given a priori. The corresponding discrete variable is

$$
g_{p}:=\int_{\Gamma_{C}^{s}} g \phi_{p} d s
$$

Now, for Tresca friction conditions we have to replace the friction bound $\mathfrak{F}\left|\lambda_{n, p, s}\right|$ in (2.8) by $g_{p}$.

\section{Treatment of Tresca friction in 3D}

In this section, we derive our algorithm for the treatment of friction in the three-dimensional case. To simplify the presentation, we restrict ourselves to the case $u_{n, p}=0$ for all nodes $p \in \mathcal{S}$, with Tresca friction. In the following sections, we will return to the more general Signorini contact condition (2.3) combined with Coulomb friction. We remark that due to the discussion in Subsection 2.2, the following part holds as well for a one-body contact problem as for a two-body contact problem.

In our iterative algorithm, we have to solve in each iteration step a linear problem with boundary conditions of Dirichlet-, Neumann- or Robin-type on the contact zone $\Gamma_{C}$. The detection of the zones for the different types of boundary conditions is based on a primal-dual approach. The Lagrange multiplier playing the role of the dual variable can be locally computed in a postprocess from the primal variable $\boldsymbol{u}_{h}$, see (2.6). This algorithm for 3D friction is closely related to the primal-dual active set method for $2 \mathrm{D}$ friction. Consequently, it perfectly fits into the abstract framework used in [HW05, 
HMW06] and inherits the advantages of these methods, i.e., their simple implementation and their numerical efficiency. The method can be regarded as an active set strategy or alternatively as a semismooth Newton method. Our derivation below is based on the latter aspect that also guarantees fast local convergence of the iterates, see Theorem 4.1.

3.1. Derivation of the iteration scheme. To start with, we review the conditions for 3D Tresca friction as given by (2.8) using $g_{p}$ instead of $\mathfrak{F}\left|\lambda_{n, p, s}\right|$. Since for $g_{p}=0$ these conditions simplify to homogeneous Neumann boundary conditions in tangential direction, we first assume that $g_{p}>0$ and, on page 13 comment on the case $g_{p}=0$. By a straightforward calculation, it can be verified that (2.8) with $g_{p}$ instead of $\mathfrak{F}\left|\lambda_{n, p, s}\right|$ is equivalent to $\mathcal{C}\left(\boldsymbol{u}_{\tau, p}, \boldsymbol{\lambda}_{\tau, p, s}\right)=0$ for all $p \in \mathcal{S}, c_{\tau}>0$, where the nonlinear complementarity function $\mathcal{C}(\cdot, \cdot)$ is defined by

$$
\mathcal{C}\left(\boldsymbol{u}_{\tau, p}, \boldsymbol{\lambda}_{\tau, p, s}\right):=\max \left(g_{p},\left\|\boldsymbol{\lambda}_{\tau, p, s}+c_{\tau} \boldsymbol{u}_{\tau, p}\right\|\right) \boldsymbol{\lambda}_{\tau, p, s}-g_{p}\left(\boldsymbol{\lambda}_{\tau, p, s}+c_{\tau} \boldsymbol{u}_{\tau, p}\right)
$$

We remark that the equivalence between (2.8) with $g_{p}$ instead of $\mathfrak{F}\left|\lambda_{n, p, s}\right|$ and (3.1) and the results to be presented below hold for each $c_{\tau}>0$.

In the sequel, we take (3.1) as the starting point for our algorithm. Our main idea is, to apply a Newton-type algorithm for the solution of $\mathcal{C}\left(\boldsymbol{u}_{\tau, p}, \boldsymbol{\lambda}_{\tau, p, s}\right)=$ 0 . Unfortunately, both the Euclidean norm and the max-function are not smooth. However, they are semismooth in the sense of [QS93, HIK03] which justifies the application of a semismooth Newton method. As generalized derivative for $f(b):=\max (a, b)$, we use $\mathcal{G}_{f}(b)=0$ if $a \geq b$ and $\mathcal{G}_{f}(b)=1$ if $a<b$. We remark that the arbitrary choice for the case $a=b$ does not influence the convergence rate of the proposed algorithm. Note that, in the first term of (3.1) the Euclidean norm appears for nonzero arguments only. This is due to the fact that if $\left\|\boldsymbol{\lambda}_{\tau, p, s}+c_{\tau} \boldsymbol{u}_{\tau, p}\right\|=0$, we obtain $\max \left(g_{p},\left\|\boldsymbol{\lambda}_{\tau, p, s}+c_{\tau} \boldsymbol{u}_{\tau, p}\right\|\right)=g_{p}$ and the Euclidean norm vanishes. Therefore, the only non-differentiability that matters in (3.1) is the max-function. In the semismooth Newton step the derivative of the Euclidean norm only occurs for points that are differentiable in the classical sense. 
We now compute, for $p \in \mathcal{S}$, the generalized derivative $\mathcal{G}_{C}$ of $\mathcal{C}(\cdot, \cdot)$. For the variation $\left(\delta \boldsymbol{u}_{\tau, p}, \delta \boldsymbol{\lambda}_{\tau, p, s}\right) \in \mathbb{R}^{2} \times \mathbb{R}^{2}$, we obtain

$$
\begin{aligned}
& \mathcal{G}_{C}\left(\boldsymbol{u}_{\tau, p}, \boldsymbol{\lambda}_{\tau, p, s}\right)\left(\delta \boldsymbol{u}_{\tau, p}, \delta \boldsymbol{\lambda}_{\tau, p, s}\right)=\max \left(g_{p},\left\|\boldsymbol{\lambda}_{\tau, p, s}+c_{\tau} \boldsymbol{u}_{\tau, p}\right\|\right) \delta \boldsymbol{\lambda}_{\tau, p, s} \\
& \quad+\chi_{\mathcal{A}} \frac{\boldsymbol{\lambda}_{\tau, p, s}\left(\boldsymbol{\lambda}_{\tau, p, s}+c_{\tau} \boldsymbol{u}_{\tau, p}\right)^{\top}}{\left\|\boldsymbol{\lambda}_{\tau, p, s}+c_{\tau} \boldsymbol{u}_{\tau, p}\right\|}\left(\delta \boldsymbol{\lambda}_{\tau, p, s}+c_{\tau} \delta \boldsymbol{u}_{\tau, p}\right)-g_{p}\left(\delta \boldsymbol{\lambda}_{\tau, p, s}+c_{\tau} \delta \boldsymbol{u}_{\tau, p}\right) .
\end{aligned}
$$

Here, $\chi_{\mathcal{A}}$ denotes the characteristic function of the set $\mathcal{A}_{\tau}=\left\{p \in \mathcal{S}: \| \boldsymbol{\lambda}_{\tau, p, s}+\right.$ $c_{\tau} \boldsymbol{u}_{\tau, p} \|>g_{p}$ \}, i.e., $\chi_{\mathcal{A}}=1$ if $\left\|\boldsymbol{\lambda}_{\tau, p, s}+c_{\tau} \boldsymbol{u}_{\tau, p}\right\|>g_{p}$, and $\chi_{\mathcal{A}}=0$ if $\| \boldsymbol{\lambda}_{\tau, p, s}+$ $c_{\tau} \boldsymbol{u}_{\tau, p} \| \leq g_{p}$. We note that $\boldsymbol{\lambda}_{\tau, p, s}\left(\boldsymbol{\lambda}_{\tau, p, s}+c_{\tau} \boldsymbol{u}_{\tau, p}\right)^{\top}$ is a $2 \times 2$-matrix, either zero or of rank one. Performing a semismooth Newton step at a current iterate $\left(\boldsymbol{u}_{\tau, p}^{k-1}, \boldsymbol{\lambda}_{\tau, p, s}^{k-1}\right)$, one derives the new iterates $\left(\boldsymbol{u}_{\tau, p}^{k}, \boldsymbol{\lambda}_{\tau, p, s}^{k}\right)$ from

Solve $\mathcal{G}_{C}\left(\boldsymbol{u}_{\tau, p}^{k-1}, \boldsymbol{\lambda}_{\tau, p, s}^{k-1}\right)\left(\delta \boldsymbol{u}_{\tau, p}^{k}, \delta \boldsymbol{\lambda}_{\tau, p, s}^{k}\right)=-\mathcal{C}\left(\boldsymbol{u}_{\tau, p}^{k-1}, \boldsymbol{\lambda}_{\tau, p, s}^{k-1}\right)$

and update

$$
\left(\boldsymbol{u}_{\tau, p}^{k}, \boldsymbol{\lambda}_{\tau, p, s}^{k}\right)=\left(\boldsymbol{u}_{\tau, p}^{k-1}, \boldsymbol{\lambda}_{\tau, p, s}^{k-1}\right)+\left(\delta \boldsymbol{u}_{\tau, p}^{k}, \delta \boldsymbol{\lambda}_{\tau, p, s}^{k}\right) .
$$

The characteristic function $\chi_{\mathcal{A}}$ in (3.2) separates the nodes of $\mathcal{S}$ into $\mathcal{I}_{\tau}^{k}$, the inactive set, and $\mathcal{A}_{\tau}^{k}$, the active set, according to

$$
\begin{aligned}
\mathcal{I}_{\tau}^{k} & :=\left\{p \in \mathcal{S}:\left\|\boldsymbol{\lambda}_{\tau, p, s}^{k-1}+c_{\tau} \boldsymbol{u}_{\tau, p}^{k-1}\right\|-g_{p} \leq 0\right\}, \\
\mathcal{A}_{\tau}^{k} & :=\left\{p \in \mathcal{S}:\left\|\boldsymbol{\lambda}_{\tau, p, s}^{k-1}+c_{\tau} \boldsymbol{u}_{\tau, p}^{k-1}\right\|-g_{p}>0\right\} .
\end{aligned}
$$

Using this notation and (3.2) in (3.3), a straightforward computation shows that the new iterate $\left(\boldsymbol{u}_{\tau, p}^{k}, \boldsymbol{\lambda}_{\tau, p, s}^{k}\right)$ satisfies

$$
\begin{aligned}
\boldsymbol{u}_{\tau, p}^{k}=0 & \text { for } p \in \mathcal{I}_{\tau}^{k}, \\
\left(I d_{2}-M_{p}^{k-1}\right) \boldsymbol{\lambda}_{\tau, p, s}^{k}-c_{\tau} M_{p}^{k-1} \boldsymbol{u}_{\tau, p}^{k}=\boldsymbol{h}_{p}^{k-1} & \text { for } p \in \mathcal{A}_{\tau}^{k},
\end{aligned}
$$

where $M_{p}^{k-1}:=e_{p}^{k-1}\left(I d_{2}-F_{p}^{k-1}\right)$, with the scalar value $e_{p}^{k-1}$ and the $2 \times 2$ matrix $F_{p}^{k-1}$ given by

$$
e_{p}^{k-1}:=\frac{g_{p}}{\left\|\boldsymbol{\lambda}_{\tau, p, s}^{k-1}+c_{\tau} \boldsymbol{u}_{\tau, p}^{k-1}\right\|}, \quad F_{p}^{k-1}:=\frac{\boldsymbol{\lambda}_{\tau, p, s}^{k-1}\left(\boldsymbol{\lambda}_{\tau, p, s}^{k-1}+c_{\tau} \boldsymbol{u}_{\tau, p}^{k-1}\right)^{\top}}{g_{p}\left\|\boldsymbol{\lambda}_{\tau, p, s}^{k-1}+c_{\tau} \boldsymbol{u}_{\tau, p}^{k-1}\right\|},
$$

and the vector $\boldsymbol{h}_{p}^{k-1} \in \mathbb{R}^{2}$

$$
\boldsymbol{h}_{p}^{k-1}:=e_{p}^{k-1} F_{p}^{k-1}\left(\boldsymbol{\lambda}_{\tau, p, s}^{k-1}+c_{\tau} \boldsymbol{u}_{\tau, p}^{k-1}\right) .
$$


Note that, while on the inactive set $\mathcal{I}_{\tau}^{k}$ Dirichlet conditions are imposed, the condition on the active set $\mathcal{A}_{\tau}^{k}$ is of Robin-type since it involves the displacement $\boldsymbol{u}_{\tau, p}^{k}$ and the surface traction $\boldsymbol{\lambda}_{\tau, p, s}^{k}$. The sets $\mathcal{A}_{\tau}^{k}$ and $\mathcal{I}_{\tau}^{k}$ approximate the sets of slippy and of sticky nodes at the solution, respectively. In the more general setting of Signorini conditions, we have also to set the boundary condition in normal direction. The Robin condition (3.5b) can be easily handled if we rewrite $(3.5 \mathrm{~b})$ as

$$
-\boldsymbol{\lambda}_{\tau, p, s}^{k}+L_{p}^{k-1} \boldsymbol{u}_{\tau, p}^{k}=\boldsymbol{r}_{p}^{k-1} .
$$

We note that $\boldsymbol{r}_{p}^{k-1}$ enters in the right hand side of the linear system and $L_{p}^{k-1}$ gives a contribution to the system matrix. Comparing (3.8) with (3.5b) and (3.7), we get under the assumption that $I d_{2}-M_{p}^{k-1}$ is regular

$$
\begin{aligned}
L_{p}^{k-1} & :=c_{\tau}\left(I d_{2}-M_{p}^{k-1}\right)^{-1} M_{p}^{k-1}=c_{\tau}\left(\left(I d_{2}-M_{p}^{k-1}\right)^{-1}-I d_{2}\right), \\
\boldsymbol{r}_{p}^{k-1} & :=-\left(I d_{2}-M_{p}^{k-1}\right)^{-1} \boldsymbol{h}_{p}^{k-1} .
\end{aligned}
$$

We mention that this Robin condition only guarantees positive definiteness of the system matrix if $L_{p}^{k-1}$ is positive definite. Note that for $L_{p}^{k-1}=0$, we find a pure Neumann condition. The degeneration of Robin type to Dirichlet type boundary conditions is not included in the form (3.9). However, this is not necessary for our problem, since nodes $p$ with a Dirichlet condition belong to the set $\mathcal{A}_{\tau}^{k}$ and therefore are not handled by (3.8). Obviously one can see that the matrix $I d_{2}-M_{p}^{k-1}$ is not necessary regular and therefore not invertible. In the next subsection, we are going to present three possible modifications of the Robin system (3.5b) such that a regular matrix $I d_{2}-M_{p}^{k-1}$ is obtained. Two of these modifications give a positive definite and symmetric matrix $L_{p}^{k-1}$. We remark that all modifications converge in the limit case to the original system (3.5b) and thus preserves the local convergence properties of the numerical algorithm.

3.2. Modifications of the Robin system. To obtain a robust and convergent scheme, we have to replace the matrix $F_{p}^{k-1}$ by a scaled matrix $\tilde{F}_{p, l}^{k-1}$, $l=1,2,3$. The index $l$ stands for one of the three possibilities considered in this paper. The scaling can be seen as a projection of the Lagrange multiplier onto its feasible set given in (2.8). We remark that our numerical experience shows that this scaling is essential for the robustness of the iteration scheme. According to the definition of $M_{p}^{k-1}$ and (3.7), we replace in (3.5b) $M_{p}^{k-1}$ by 
$\tilde{M}_{p, l}^{k-1}$ and $\boldsymbol{h}_{p}^{k-1}$ by $\tilde{\boldsymbol{h}}_{p, l}^{k-1}$ given by

$\tilde{M}_{p, l}^{k-1}:=e_{p}^{k-1}\left(I d_{2}-\tilde{F}_{p, l}^{k-1}\right), \quad \tilde{\boldsymbol{h}}_{p, l}^{k-1}:=e_{p}^{k-1} \tilde{F}_{p, l}^{k-1}\left(\boldsymbol{\lambda}_{\tau, p, s}^{k-1}+c_{\tau} \boldsymbol{u}_{\tau, p}^{k-1}\right), \quad l=1,2,3$.

In a second step, we replace the matrix $I d_{2}-M_{p}^{k-1}$ by $I d_{2}-\beta_{p, l}^{k-1} \tilde{M}_{p, l}^{k-1}$ with a scaling factor $\beta_{p, l}^{k-1}>0$, such that the resulting matrix is regular. Then the form (3.8) of the Robin boundary conditions is written as $-\boldsymbol{\lambda}_{\tau, p, s}^{k}+\tilde{L}_{p, l}^{k-1} \boldsymbol{u}_{\tau, p}^{k}=$ $\tilde{\boldsymbol{r}}_{p, l}^{k-1}$ with

$\tilde{L}_{p, l}^{k-1}:=c_{\tau}\left(\left(I d_{2}-\beta_{p, l}^{k-1} \tilde{M}_{p, l}^{k-1}\right)^{-1}-I d_{2}\right), \quad \tilde{\boldsymbol{r}}_{p, l}^{k-1}:=-\left(I d_{2}-\beta_{p, l}^{k-1} \tilde{M}_{p, l}^{k-1}\right)^{-1} \tilde{\boldsymbol{h}}_{p, l}^{k-1}$

for $l=1,2,3$. We mention that similar modifications as done for the matrix $F_{p}^{k-1}$ are used in primal-dual algorithms for the minimization of functionals involving Euclidean norms, see, e.g., [ACCO00, CGM99, HS06]). These type of problems have a similar structure as (3.5). Clearly, this relation is due to the fact that the Tresca friction problem also corresponds to the minimization of a nonsmooth energy functional.

Since for all modifications $\tilde{F}_{p, l}^{k-1} \rightarrow F_{p}^{k-1}$ and $\beta_{p, l}^{k-1} \rightarrow 1$ as $\left(\boldsymbol{u}_{\tau, p}^{k}, \boldsymbol{\lambda}_{\tau, p, s}^{k}\right)$ converges to the solution the modifications do not degrade the local superlinear convergence. Next we present the three possible modifications used in this paper.

First modification. We use a parameter $\beta_{p, 1}^{k-1} \neq 1$ in $I d_{2}-\beta_{p, 1}^{k-1} M_{p}$ in (3.10) only if $\left(\boldsymbol{\lambda}_{\tau, p, s}^{k-1}\right)^{\top}\left(\boldsymbol{\lambda}_{\tau, p, s}^{k-1}+c_{\tau} \boldsymbol{u}_{\tau, p}^{k-1}\right)<0$. This condition is equivalent to the fact that the angle between the two vectors is greater than 90 degree. Since in the limit case both vectors are parallel, the modification only applies when the iterates are far away from the solution. In this modification, the unsymmetric matrix $L_{p}^{k-1}$ is replaced by $\tilde{L}_{p, 1}^{k-1}$, which is unsymmetric as well. We project the Lagrange multiplier onto its feasible set and define

$$
\tilde{F}_{p, 1}^{k-1}:=\frac{\boldsymbol{\lambda}_{\tau, p, s}^{k-1}\left(\boldsymbol{\lambda}_{\tau, p, s}^{k-1}+c_{\tau} \boldsymbol{u}_{\tau, p}^{k-1}\right)^{\top}}{\max \left(g_{p},\left\|\boldsymbol{\lambda}_{\tau, p, s}^{k-1}\right\|\right)\left\|\boldsymbol{\lambda}_{\tau, p, s}^{k-1}+c_{\tau} \boldsymbol{u}_{\tau, p}^{k-1}\right\|} .
$$

Using

$$
\alpha_{p}^{k-1}:=\frac{\left(\boldsymbol{\lambda}_{\tau, p, s}^{k-1}\right)^{\top}\left(\boldsymbol{\lambda}_{\tau, p, s}^{k-1}+c_{\tau} \boldsymbol{u}_{\tau, p}\right)}{\left\|\boldsymbol{\lambda}_{\tau, p, s}^{k-1}\right\|\left\|\boldsymbol{\lambda}_{\tau, p, s}^{k-1}+c_{\tau} \boldsymbol{u}_{\tau, p}\right\|}, \quad \delta_{p}^{k-1}:=\min \left\{\frac{\left\|\boldsymbol{\lambda}_{\tau, p, s}^{k-1}\right\|}{g_{p}}, 1\right\}
$$


it is easy to see that $\gamma_{\tilde{F}_{p, 1}}^{1}=\alpha_{p}^{k-1} \delta_{p}^{k-1}$ is an eigenvalue of the matrix $\tilde{F}_{p, 1}^{k-1}$ with the eigenvector $\boldsymbol{\lambda}_{\tau, p, s}^{k-1}$. The second eigenvalue is given by $\gamma_{\tilde{F}_{p, 1}}^{2}=0$ where the corresponding eigenvector is the vector which is orthogonal to $\boldsymbol{\lambda}_{\tau, p, s}^{k-1}+c_{\tau} \boldsymbol{u}_{\tau, p}^{k-1}$. Therefore, the eigenvalues of $\tilde{M}_{p, 1}^{k-1}$ are $\gamma_{\tilde{M}_{p, 1}}^{1}=e_{p}^{k-1}\left(1-\alpha_{p}^{k-1} \delta_{p}^{k-1}\right)$ and $\gamma_{\tilde{M}_{p, 1}}^{2}=$ $e_{p}^{k-1}$. Since $-1 \leq \alpha_{p}^{k-1} \leq 1$ and $0 \leq \delta_{p}^{k-1} \leq 1$, we get due to $0<e_{p}^{k-1}<1$ the relation $0 \leq \gamma_{\tilde{M}_{p, 1}}^{1}<2$ and $0<\gamma_{\tilde{M}_{p, 1}}^{2}<1$. Using (3.10) with

$$
\beta_{p, 1}^{k-1}:=\left\{\begin{array}{cl}
\frac{1}{1-\alpha_{p}^{k-1} \delta_{p}^{k-1}} & \text { if } \alpha_{p}^{k-1}<0 \\
1 & \text { else. }
\end{array}\right.
$$

yields a unsymmetric matrix $\tilde{L}_{p, 1}^{k-1}$ with positive eigenvalues.

Second modification. In contrast to the first modification, we use a symmetric matrix $\tilde{F}_{p, 2}^{k-1}$. Here we need a parameter $\beta_{p, 2}^{k-1} \neq 1$ in (3.10) for all cases with $\left(\boldsymbol{\lambda}_{\tau, p, s}^{k-1}\right)^{\top}\left(\boldsymbol{\lambda}_{\tau, p, s}^{k-1}+c_{\tau} \boldsymbol{u}_{\tau, p}^{k-1}\right) \neq 0$. We replace $F_{p}^{k-1}$ by the symmetrization of (3.11), namely

$$
\tilde{F}_{p, 2}^{k-1}:=\frac{\boldsymbol{\lambda}_{\tau, p, s}^{k-1}\left(\boldsymbol{\lambda}_{\tau, p, s}^{k-1}+c_{\tau} \boldsymbol{u}_{\tau, p}^{k-1}\right)^{\top}+\left(\boldsymbol{\lambda}_{\tau, p, s}^{k-1}+c_{\tau} \boldsymbol{u}_{\tau, p}^{k-1}\right)\left(\boldsymbol{\lambda}_{\tau, p, s}^{k-1}\right)^{\top}}{2 \max \left(g_{p},\left\|\boldsymbol{\lambda}_{\tau, p, s}^{k-1}\right\|\right)\left\|\boldsymbol{\lambda}_{\tau, p, s}^{k-1}+c_{\tau} \boldsymbol{u}_{\tau, p}^{k-1}\right\|} .
$$

One can proof that the eigenvalues of $\tilde{F}_{p, 2}^{k-1}$ are $\gamma_{\tilde{F}_{p, 2}^{1,2}}^{1, p}=\frac{1}{2}\left(\alpha_{p}^{k-1} \pm 1\right) \delta_{p}^{k-1} \in$ $[-1,1]$ and therefore the eigenvalues of the matrix $\tilde{M}_{p, 2}^{k-1}$ are $\gamma_{\tilde{M}_{p, 2}^{1,2}}^{1,2}=e_{p}^{k-1}(2-$ $\left.\left(\alpha_{p}^{k-1} \pm 1\right) \delta_{p}^{k-1}\right) / 2$. Using the same arguments as before we get $0 \leq \gamma_{\tilde{M}_{p, 2}}^{1,2}<2$. Setting

$$
\beta_{p, 2}^{k-1}:=\frac{2}{2-\left(\alpha_{p}^{k-1}-1\right) \delta_{p}^{k-1}}
$$

results in a symmetric and positive definite matrix $\tilde{L}_{p, 2}^{k-1}$.

Third modification. In the third modification, we use the matrix

$$
\tilde{F}_{p, 3}^{k-1}:=\frac{\boldsymbol{\lambda}_{\tau, p, s}^{k-1}\left(\boldsymbol{\lambda}_{\tau, p, s}^{k-1}\right)^{\top}}{\max \left(g_{p},\left\|\boldsymbol{\lambda}_{\tau, p, s}^{k-1}\right\|\right)^{2}}
$$

instead of $F_{p}^{k-1}$. Obviously this matrix is symmetric and positive semidefinite with eigenvalues $\gamma_{\tilde{F}_{p, 3}}^{1}=0$ and $\gamma_{\tilde{F}_{p, 3}}^{2}=\left(\delta_{p}^{k-1}\right)^{2}$. Therefore we get for the eigenvalues of the matrix $\tilde{M}_{p, 3}^{k-1}$ due to $0<e_{p}^{k-1}<1$ the relation $0 \leq \gamma_{\tilde{M}_{p, 3}}<1$ 
and the matrix $\tilde{L}_{p, 3}^{k-1}$ defined by (3.10) with $\beta_{p, 3}^{k-1}=1$ is symmetric and positive definite. We remark that the matrix $\tilde{F}_{p, 3}^{k-1}$ converges in the limit case to the matrix $F_{p}^{k-1}$ since in the solution for a node $p \in \mathcal{A}_{\tau}$, we have

$$
\frac{\boldsymbol{\lambda}_{\tau, p, s}+c_{\tau} \boldsymbol{u}_{\tau, p}}{\left\|\boldsymbol{\lambda}_{\tau, p, s}+c_{\tau} \boldsymbol{u}_{\tau, p}\right\|}=\frac{\boldsymbol{\lambda}_{\tau, p, s}}{g_{p}}
$$

3.3. Extension to the case $g_{p}=0$. In the above discussion, we assumed $g_{p}>0$ for all $p \in \mathcal{S}$. The reason for this assumption is that for $g_{p}=0$ one cannot stringently deduce $\boldsymbol{\lambda}_{\tau, p, s}=0$ (which follows directly from (2.8)) from $\mathcal{C}\left(\boldsymbol{u}_{\tau, p}, \boldsymbol{\lambda}_{\tau, p, s}\right)=0$. However, if Tresca friction combined with fixed point ideas is used to solve Coulomb friction problems, $g_{p}=0$ naturally occurs for all non-contact points, which makes the case $g_{p}=0$ rather important. Fortunately, after some minor changes, nodes $p$ with $g_{p}=0$ can also be handled using (3.5) with (3.11)-(3.13).

In the following, we consider $p \in \mathcal{S}$ with $g_{p}=0$. First, we assume $\| \boldsymbol{\lambda}_{\tau, p, s}^{k-1}+$ $c_{\tau} \boldsymbol{u}_{\tau, p}^{k-1} \|>0$. Then, $p \in \mathcal{A}_{\tau}^{k}$ since $g_{p}=0$. In the case $\left\|\boldsymbol{\lambda}_{\tau, p, s}^{k-1}+c_{\tau} \boldsymbol{u}_{\tau, p}^{k-1}\right\|=0$ we have to set $p \in \mathcal{A}_{\tau}^{k}$. In both cases, we set $\tilde{M}_{p, l}^{k-1}=0$ and (3.5b) leads to the desired homogeneous Neumann condition $\boldsymbol{\lambda}_{\tau, p, s}^{k}=0$. We mention that in the case $\left\|\boldsymbol{\lambda}_{\tau, p, s}^{k-1}+c_{\tau} \boldsymbol{u}_{\tau, p}^{k-1}\right\|>0$ and $\left\|\boldsymbol{\lambda}_{\tau, p, s}^{k-1}\right\|>0$, we get due to (3.4b) and (3.6) automatically $p \in \mathcal{A}_{\tau}^{k}$ and $e_{p}^{k-1}=0$ and therefore $\tilde{M}_{p, l}^{k-1}=0$. In particular, these matrices are well defined for this case. So only for the cases $\left\|\boldsymbol{\lambda}_{\tau, p, s}^{k-1}+c_{\tau} \boldsymbol{u}_{\tau, p}^{k-1}\right\|=0$ or $\left\|\boldsymbol{\lambda}_{\tau, p, s}^{k-1}\right\|=0$, we have to enforce the node $p$ to be in $\mathcal{A}_{\tau}^{k-1}$.

3.4. General Remarks. Computing generalized derivatives of nonsmooth functionals is a delicate issue. While in [AC91] mainly intuitive arguments are used, the related papers [CP99, CKPS98] use the concept of Bouliganddifferentiability. This concept allows for the use of globalization (e.g., linesearch) strategies, but calculating the search direction requires to solve a nonlinear system in each Newton step. In these papers, this problem is circumvented by substituting this nonlinear system by a linear one. Despite this heuristic step, the authors report on good numerical results. The concept of semismoothness [QS93, HIK03] used in the present paper has the advantage that the search direction can be found by solving a linear system. Nevertheless, one can also proof local superlinear convergence of the iterates, see Theorem 4.1. 
Furthermore, we remark that there is some freedom in choosing the nonlinear complementarity function to express the complementarity conditions for the Tresca friction law. For $g_{p}>0$, we can also work with (3.1) by

$$
\overline{\mathcal{C}}\left(\boldsymbol{u}_{\tau, p}, \boldsymbol{\lambda}_{\tau, p, s}\right):=\boldsymbol{\lambda}_{\tau, p, s}-g_{p} \frac{\left(\boldsymbol{\lambda}_{\tau, p, s}+c_{\tau} \boldsymbol{u}_{\tau, p}\right)}{\max \left(g_{p},\left\|\boldsymbol{\lambda}_{\tau, p, s}+c_{\tau} \boldsymbol{u}_{\tau, p}\right\|\right)} .
$$

Complementarity functions closely related to (3.14) for dealing with friction conditions have been used in [AC91, CP99, CKPS98]. A semismooth Newton iteration for the solution of $\overline{\mathcal{C}}\left(\boldsymbol{u}_{\tau, p}, \boldsymbol{\lambda}_{\tau, p, s}\right)=0$ results in an iteration rule that also uses the active and inactive sets defined in (3.4), but results in a modified iteration step on $\mathcal{A}_{\tau}^{k}$. Our numerical experience yields that algorithms based on (3.1) perform more robust compared to those based on (3.14).

3.5. Algebraic representation. In this subsection, we give the matrix representation of the algebraic system, we have to solve in each iteration step. As mentioned above, we restrict ourselves to the case $u_{n, p}=0$. The tangential conditions are either Dirichlet conditions for the inactive nodes $p \in \mathcal{I}_{\tau}^{k}$ or Robin conditions for the active nodes $p \in \mathcal{A}_{\tau}^{k}$. To rotate the stiffness matrix $A$ arising form standard linear elasticity and introduced in (2.5), we define the matrix with the normal vectors by

$$
N:=\left(\begin{array}{ccccccccc} 
& & \ddots & 0 & 0 & 0 & & & \\
0 & \cdots & 0 & & \boldsymbol{n}_{p}^{\top} & & 0 & \cdots & 0 \\
& & & 0 & 0 & 0 & \ddots & &
\end{array}\right) \in \mathbb{R}^{|\mathcal{S}| \times 3|\mathcal{S}|},
$$

and the matrix with tangential vectors by

$$
T:=\left(\begin{array}{ccccccccc} 
& & \ddots & 0 & 0 & 0 & & & \\
0 & \cdots & 0 & & \boldsymbol{\tau}_{1 p}^{\top} & & 0 & \cdots & 0 \\
0 & \cdots & 0 & & \boldsymbol{\tau}_{2 p}^{\top} & & 0 & \cdots & 0 \\
& & & 0 & 0 & 0 & \ddots & &
\end{array}\right) \in \mathbb{R}^{2|\mathcal{S}| \times 3|\mathcal{S}|}
$$

where as above, for each $p \in \mathcal{S}, \boldsymbol{n}_{p}, \boldsymbol{\tau}_{1 p}$ and $\boldsymbol{\tau}_{2 p}$ denote the unit outward normal and the tangential vectors, respectively. Moreover, for $p \in \mathcal{A}_{\tau}^{k}$ the matrices $\tilde{L}_{p, l}^{k-1}$ are assembled into

$$
L_{\mathcal{A}_{\tau}^{k}}:=\operatorname{diag}\left\{\tilde{L}_{p, l}^{k-1}\right\}_{p \in \mathcal{A}_{\tau}^{k}} \in \mathbb{R}^{2\left|\mathcal{A}_{\tau}^{k}\right| \times 2\left|\mathcal{A}_{\tau}^{k}\right|} .
$$

Similarly, we define the vector $\boldsymbol{r}_{\mathcal{A}_{\tau}^{k}}$ by the entries $\tilde{\boldsymbol{r}}_{p, l}^{k-1}$ for $p \in \mathcal{A}_{\tau}^{k}$. We now use the decomposition of $\mathcal{S}$ into $\mathcal{A}_{\tau}^{k}$ and $\mathcal{I}_{\tau}^{k}$. The vector $\boldsymbol{u}_{h}$ can then be 
partitioned into $\left(\boldsymbol{u}_{\mathcal{N}}^{\top}, \boldsymbol{u}_{\mathcal{I}_{\tau}^{k}}^{\top}, \boldsymbol{u}_{\mathcal{A}_{\tau}^{k}}^{\top}\right)^{\top}$, the multiplier $\boldsymbol{\lambda}_{h}$ into $\left(\boldsymbol{\lambda}_{\mathcal{I}_{\tau}^{k}}^{\top}, \boldsymbol{\lambda}_{\mathcal{A}_{\tau}^{k}}^{\top}\right)^{\top}$ and the right hand side $\boldsymbol{f}_{h}$ into $\left(\boldsymbol{f}_{\mathcal{N}}^{\top}, \boldsymbol{f}_{\mathcal{I}^{k}}^{\top}, \boldsymbol{f}_{\mathcal{A}_{\tau}^{k}}^{\top}\right)^{\top}$. Correspondingly, we can decompose the matrices $A_{h}, N$ and $T$, and the arising linear system has the form

$$
\left(\begin{array}{ccc}
A_{\mathcal{N N}} & A_{\mathcal{N I}_{\tau}^{k}} & A_{\mathcal{N A}_{\tau}^{k}} \\
0 & I d_{\mathcal{I}^{k}} & 0 \\
0 & 0 & N_{\mathcal{A}_{\tau}^{k}} \\
T_{\mathcal{A}_{\tau}^{k}} A_{\mathcal{A}_{\tau}^{k \mathcal{N}}} & T_{\mathcal{A}_{\tau}^{k}} A_{\mathcal{A}_{\tau}^{k} \tau_{\tau}^{k}} & T_{\mathcal{A}_{\tau}^{k}} A_{\mathcal{A}_{\tau}^{k} \mathcal{A}_{\tau}^{k}}+L_{\mathcal{A}_{\tau}^{k}} T_{\mathcal{A}_{\tau}^{k}}
\end{array}\right)\left(\begin{array}{c}
\boldsymbol{u}_{\mathcal{N}}^{k} \\
\boldsymbol{u}_{\mathcal{I}_{\tau}^{k}}^{k} \\
\boldsymbol{u}_{\mathcal{A}_{\tau}^{k}}^{k}
\end{array}\right)=\left(\begin{array}{c}
\boldsymbol{f}_{\mathcal{N}} \\
0 \\
0 \\
T_{\mathcal{A}_{\tau}^{k}} \boldsymbol{f}_{\mathcal{A}_{\tau}^{k}}+\boldsymbol{r}_{\mathcal{A}_{\tau}^{k}}
\end{array}\right)
$$

We remark that the Dirichlet boundary condition $(3.5 \mathrm{a})$ on $\mathcal{I}_{\tau}^{k}$ is reflected in the second row and the Robin-type conditions for $p \in \mathcal{A}_{\tau}^{k}$, see $(3.5 \mathrm{~b})$ or (3.8), are included in the fourth row. In the case of the more general Signorini condition, we have to replace the third row by a more complex one, see [HW05].

\section{Algorithm and numerical examples for Tresca friction}

In this section, we give the inexact primal-dual algorithm based on an efficient multigrid method to solve the resulting linear problems. Furthermore, we present a numerical example to study the performance of our algorithm and compare the three modifications of Section 3.2. While in the previous section, we have derived the iteration rule for Tresca friction without taking into account Signorini contact, the algorithm presented can handle Tresca friction and Signorini contact (2.2). The discrete Signorini conditions (2.7) are realized by applying the primal-dual active set strategy on the nonlinear complementarity function

$$
\lambda_{n, p, s}-\max \left(0, \lambda_{n, p, s}+c_{n}\left(u_{n, p}-d_{p}\right)\right)=0,
$$

with $c_{n}>0$. The strategy uses, in each iteration the active and inactive sets

$$
\begin{aligned}
\mathcal{A}_{n}^{k} & :=\left\{p \in \mathcal{S}: \lambda_{n, p, s}^{k-1}+c_{n}\left(u_{n, p}^{k-1}-d_{p}\right)>0\right\}, \\
\mathcal{I}_{n}^{k} & :=\left\{p \in \mathcal{S}: \lambda_{n, p, s}^{k-1}+c_{n}\left(u_{n, p}^{k-1}-d_{p}\right) \leq 0\right\} .
\end{aligned}
$$

We next state the inexact primal-dual active set (Ipdas) strategy for contact with Tresca friction.

\section{Algorithm 1: Ipdas for contact with 3D Tresca friction}


(0) Set $k=1$, choose $c_{n}>0, c_{\tau}>0$ and $m \in \mathbb{N}$.

Initialize $\boldsymbol{u}_{h}^{0,0}$ and $\boldsymbol{\lambda}_{h}^{0}$ as an initial solution.

(1) Define the active and inactive sets by

$$
\begin{aligned}
\mathcal{A}_{n}^{k} & :=\left\{p \in \mathcal{S}: \lambda_{n, p, s}^{k-1}+c_{n}\left(u_{n, p}^{k-1, m}-d_{p}\right)>0\right\}, \\
\mathcal{I}_{n}^{k} & :=\left\{p \in \mathcal{S}: \lambda_{n, p, s}^{k-1}+c_{n}\left(u_{n, p}^{k-1, m}-d_{p}\right) \leq 0\right\}, \\
\mathcal{A}_{\tau}^{k} & :=\left\{p \in \mathcal{S}:\left\|\boldsymbol{\lambda}_{\tau, p, s}^{k-1}+c_{\tau} \boldsymbol{u}_{\tau, p}^{k-1, m}\right\|-g_{p}>0\right\}, \\
\mathcal{I}_{\tau}^{k} & :=\left\{p \in \mathcal{S}:\left\|\boldsymbol{\lambda}_{\tau, p, s}^{k-1}+c_{\tau} \boldsymbol{u}_{\tau, p}^{k-1, m}\right\|-g_{p} \geq 0\right\} .
\end{aligned}
$$

(2) For $i=1, \ldots, m$, compute

$$
\boldsymbol{u}_{h}^{k, i}=M G\left(\boldsymbol{u}_{h}^{k, i-1}, \mathcal{A}_{n}^{k}, \mathcal{I}_{n}^{k}, \mathcal{A}_{\tau}^{k}, \mathcal{I}_{\tau}^{k}, \boldsymbol{u}_{h}^{k-1, m}, \boldsymbol{\lambda}_{h}^{k-1}\right) .
$$

(3) If $\left\|\boldsymbol{u}_{h}^{k, m}-\boldsymbol{u}_{h}^{k, 0}\right\| /\left\|\boldsymbol{u}_{h}^{k, m}\right\|<\varepsilon_{u}$ stop.

(4) Compute the Lagrange multiplier as

$$
\boldsymbol{\lambda}_{h}^{k}=D^{-1}\left(\boldsymbol{f}_{\mathcal{S}}-A_{\mathcal{S} \star} \boldsymbol{u}_{h}^{k, m}\right) .
$$

(5) Set $\boldsymbol{u}_{h}^{k+1,0}=\boldsymbol{u}_{h}^{k, m}, k=k+1$ and go to step (1).

Above, we denote by $\boldsymbol{u}_{h}^{k, i}=M G\left(\boldsymbol{u}_{h}^{k, i-1}, \mathcal{A}_{n}^{k}, \mathcal{I}_{n}^{k}, \mathcal{A}_{\tau}^{k}, \mathcal{I}_{\tau}^{k}, \boldsymbol{u}_{h}^{k-1, m}, \boldsymbol{\lambda}_{h}^{k-1}\right)$ the iterate after one multigrid step for the linear system (3.15). By $A_{\mathcal{S} \star}$, we denote the rows of the stiffness matrix $A$ corresponding to the nodes in the set $\mathcal{S}$. In the case $m \in \mathbb{N}$, we solve the linear system inexactly, i.e., we update the active and inactive sets after $m$ multigrid steps. For $m=$ " $\infty$ ", we get the exact version of our algorithm. Recalling the derivation of our iteration rule as a semismooth Newton method, we obtain the following local convergence result, see [HIK03, QS93].

Theorem 4.1. For $m=" \infty "$, Algorithm 1 converges locally superlinear.

We mention that in the multigrid approach, we call the coarsest grid, on which we solve exactly, the grid on level 0 . We get the next finer grid on level $l+1$ by decomposing each element on level $l$ into 8 subelements. For all examples presented in this paper, we use a $\mathcal{W}$-cycle with three pre- and postsmoothing steps for the multigrid. As smoother, a symmetric Gauß-Seidel iteration is applied. 
4.1. Example. As a first example for a three-dimensional contact problem with Tresca friction, we consider a one-body contact problem.

The problem setting. We consider a linearly elastic cube $\Omega=[0,1]^{3}$ with material parameters $E=200$ and $\nu=0.3$ and take the $x y$-plane as the rigid obstacle which implies that the initial gap is $d=0$. The cube is subject to the Dirichlet distortion $\boldsymbol{u}^{\top}=(0,0.2,0.06-0.15 x)$ on its upper surface $[0,1]^{2} \times\{1\}$, see the left part of Figure 1. For Tresca friction, the friction bound $g$ is given a priori and does not depend on the distortion as in the case of Coulomb friction. For this problem, we choose $g=800 x y(1-x)(1-y)$. In the right of Figure 1, we show the distorted body with the effective von

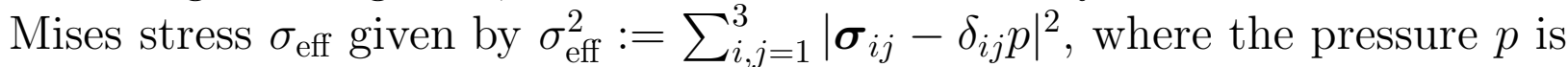
given by $p:=\frac{1}{3} \operatorname{tr}(\boldsymbol{\sigma})$.

The Tresca friction law. To get a better understanding of the different types of nodes that occur for contact problems with Tresca friction, we show in Figure 2 the nodes of the contact surface on Level 5. Different types of nodes are marked differently, see the legend in Figure 2. Note that, for each node the displacement is parallel to the multiplier $\boldsymbol{\lambda}_{\tau}$ as required. We remark that, using the Tresca friction law, nodes can stick in tangential direction without being in contact with the obstacle.
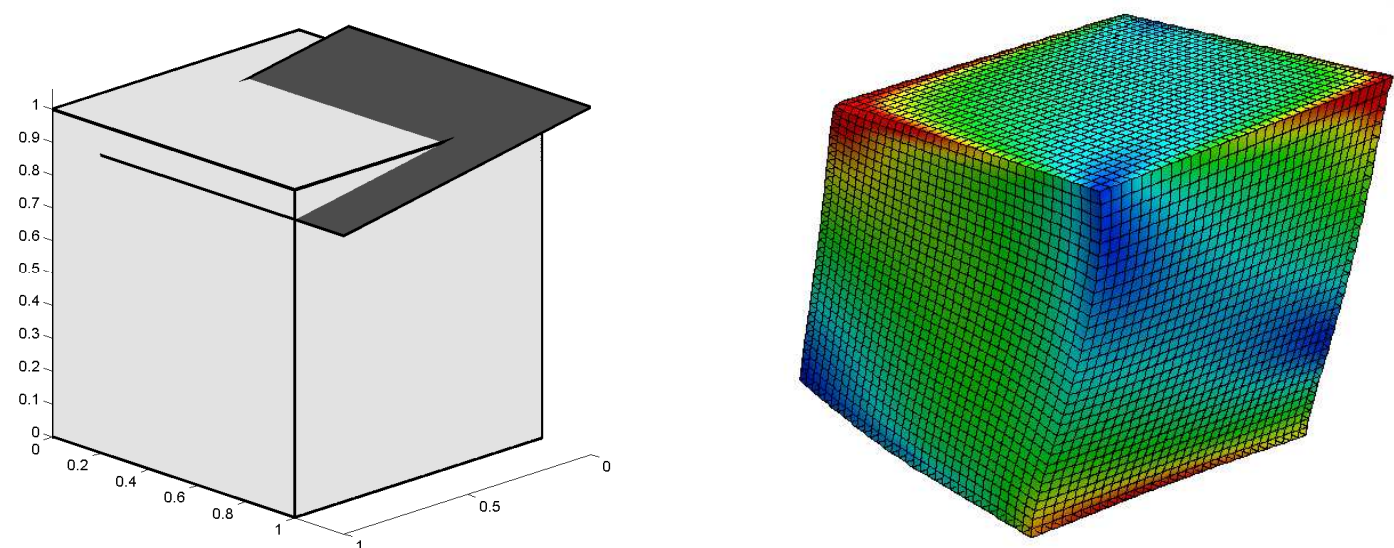

FiguRE 1. Problem definition with prescribed Dirichlet distortion (left) and distorted body with effective von Mises stress $\sigma_{\text {eff }}$; the lower surface in the plot is subject to unilateral contact and Tresca friction.

Performance of Algorithm 1. To investigate the performance of the algorithm, we first solve the linear system arising in each iteration step exactly. 

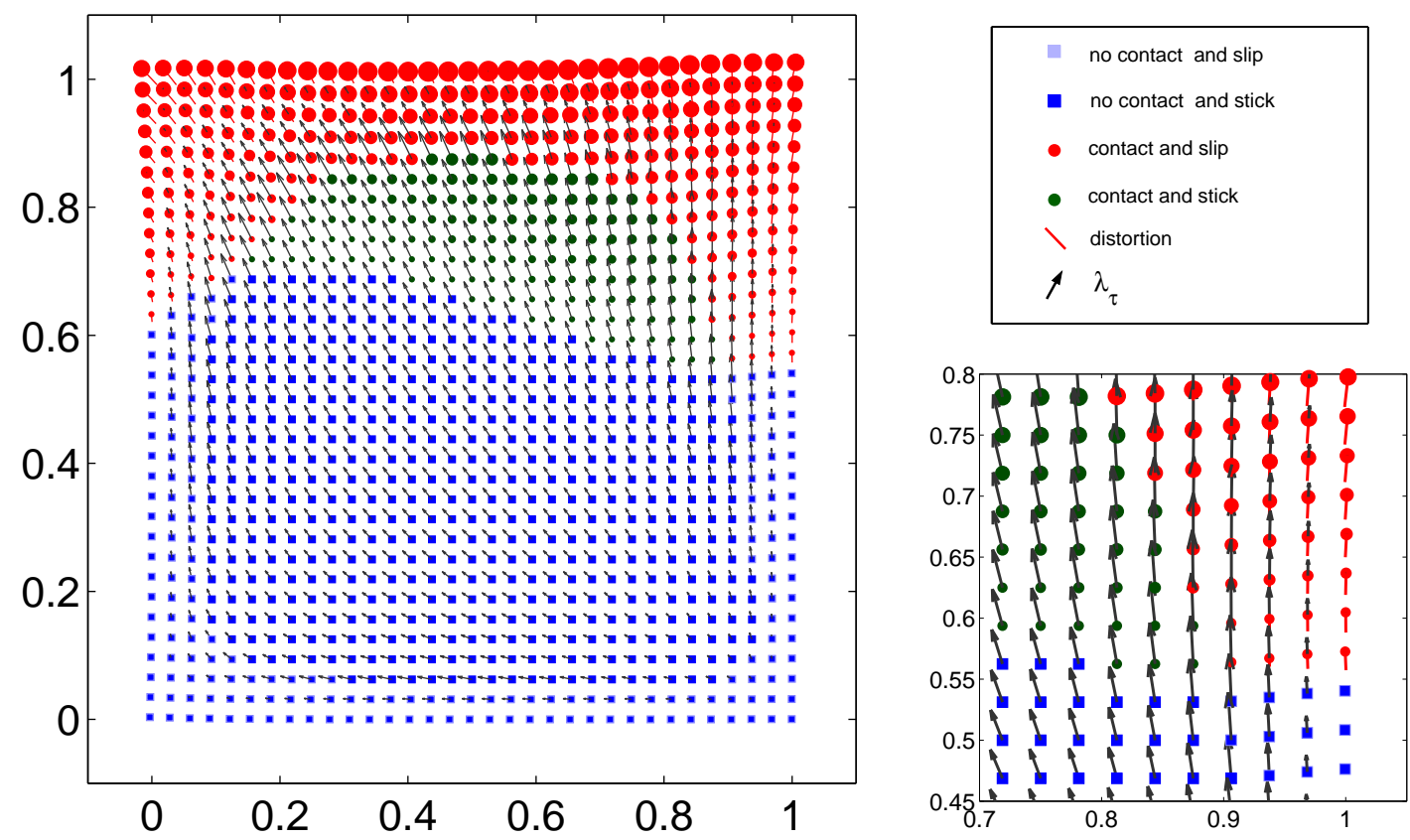

FIgURE 2. Visualization of the solution at the nodes $p \in \mathcal{S}$ for Tresca friction on Level 5 (left), legend (upper right) and cutout (lower right).

We note that in each step, our algorithm updates the contact/non-contact sets $\mathcal{A}_{n}^{k}$ and $\mathcal{I}_{n}^{k}$ as well as the slip/stick sets $\mathcal{A}_{\tau}^{k}$ and $\mathcal{I}_{\tau}^{k}$ and at the same time perform a Newton step to adopt the distortion's direction $\boldsymbol{u}_{\tau}$ to the direction of $\boldsymbol{\lambda}_{\tau}$ for sliding nodes.

TABLE 1. Performance of Algorithm 1 for the exact strategy $(m=" \infty ")$. Initialization: $\boldsymbol{u}_{h}^{0,0}=\mathbf{0}, \boldsymbol{\lambda}_{h}^{0}=\mathbf{0}$ for all levels. Tolerance $\varepsilon_{u}=10^{-9}$ with the modification (3.11).

\begin{tabular}{c||c|c|c|c|c|c|c|c}
\hline \multicolumn{1}{l||}{$\mid$} & \multicolumn{7}{c}{$\left|\mathcal{A}_{n}^{k}\right| /\left|\mathcal{A}_{\tau}^{k}\right|$ for $k=2,3,4,5,6,7,8,9$} & \\
\hline \hline 0 & $2 / 0$ & $2 / 0$ & & & & & & \\
\hline 1 & $6 / 4$ & $5 / 8$ & $5 / 8$ & $5 / 8$ & $5 / 8$ & $5 / 8$ & & \\
\hline 2 & $15 / 16$ & $12 / 16$ & $12 / 16$ & $12 / 16$ & $12 / 16$ & & & \\
\hline 3 & $49 / 32$ & $38 / 37$ & $33 / 39$ & $33 / 39$ & $33 / 39$ & $33 / 39$ & & \\
\hline 4 & $171 / 66$ & $132 / 102$ & $116 / 118$ & $112 / 122$ & $112 / 123$ & $112 / 123$ & $112 / 123$ & \\
\hline 5 & $651 / 252$ & $506 / 357$ & $443 / 411$ & $412 / 435$ & $406 / 444$ & $406 / 446$ & $406 / 446$ & $406 / 446$ \\
\hline \hline
\end{tabular}

For our tests, we initialize the algorithm on each level with $\boldsymbol{u}_{h}^{0,0}=\mathbf{0}$ and $\boldsymbol{\lambda}_{h}^{0}=\mathbf{0}$. This leads to $\mathcal{A}_{n}^{1}=\mathcal{A}_{\tau}^{1}=\emptyset$. We terminate the Ipdas-iteration if the relative change in the solution is less than $10^{-9}$. In the complementarity function, we use $c_{n}=c_{\tau}=100$; Algorithm 1 yields a fast and stable converge 

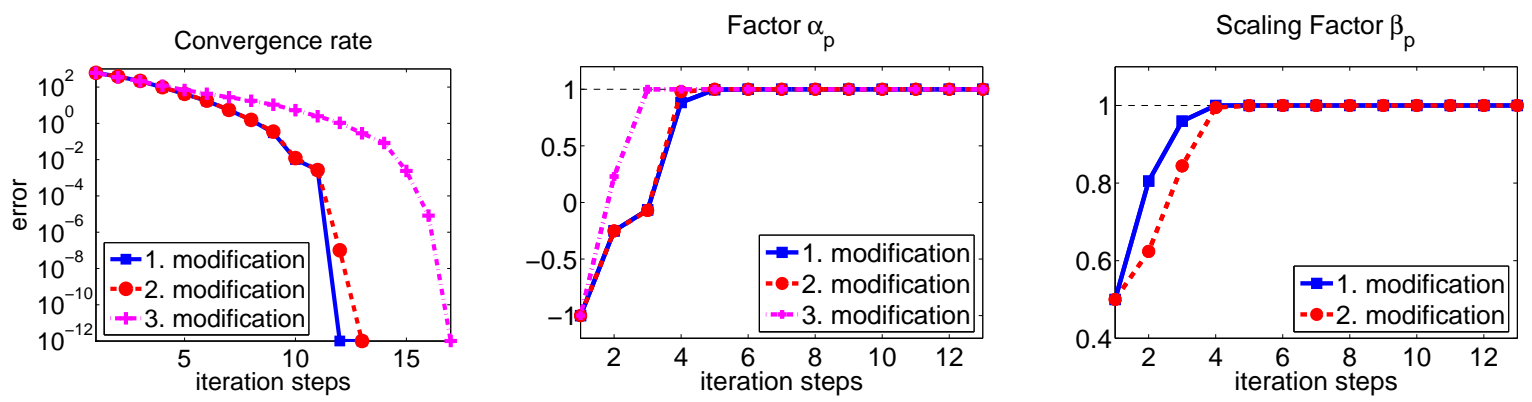

Figure 3. Convergence of $\boldsymbol{\lambda}_{h}^{k}$ on Level $5, c_{n}=c_{\tau}=100$ and $\varepsilon_{u}=10^{-14}$ (left); Behavior of $\alpha_{p, l}^{k-1}$ (middle) and $\beta_{p, l}^{k-1}$ (right) at the node $(0.0625,1,0)^{\top} \in \mathcal{S} ; \quad \boldsymbol{u}_{h}^{0,0}=(1,1,0)^{\top}, \quad \boldsymbol{\lambda}_{h}^{0}=$ $(-1,-1,0)^{\top}$.

on all levels. Table 1 shows the number of iterations needed on different refinement levels, and the number of nodes belonging to the active sets $\mathcal{A}_{n}^{k}$ and $\mathcal{A}_{\tau}^{k}$ for the first modification (3.11). We remark that for the second (3.12) and the third modification (3.13) only minor differences occur. Note that in each iteration step one linear system has to be solved. The number of iterations increases only weakly on finer levels; it seems to depend linearly on the level. Usually, after the exact active sets for both friction and contact condition are found, the method requires about 3-4 more iterations to converge. In these steps, the algorithm adjusts, for $p \in \mathcal{S}$, the direction of the tangential traction $\boldsymbol{\lambda}_{\tau, p}$ to the tangential displacement $\boldsymbol{u}_{\tau, p}$.

Comparison between the modifications. Now we compare the convergence and the behavior of the factors $\alpha_{p}^{k-1}$ and $\beta_{p, l}^{k-1}$ for the three modifications. In the left of Figure 3, we show the errors $\left\|\boldsymbol{\lambda}_{h}^{k}-\boldsymbol{\lambda}_{h}^{\star}\right\|$ on Level 5 in a logarithmic plot for the initialization $\boldsymbol{u}_{h}^{0,0}=(1,1,0)^{\top}$ and $\boldsymbol{\lambda}_{h}^{0}=(-1,-1,0)^{\top}$. Here, $\boldsymbol{\lambda}_{h}^{\star}$ denotes the Lagrange multiplier of the solution. We remark that additional iteration steps, compared to Table 1 , are needed due to the different initialization and the smaller tolerance $\varepsilon_{u}=10^{-14}$. In the middle of Figure 3, we present the cosinus $\alpha_{p}^{k-1}$ of the angle between the vectors $\boldsymbol{\lambda}_{\tau, p, s}^{k-1}$ and $\boldsymbol{\lambda}_{\tau, p, s}^{k-1}+c_{\tau} \boldsymbol{u}_{\tau, p}$ and on the right the behavior of the scaling factor $\beta_{p, l}^{k-1}$, $l=1,2$, for the node $p=(0.0625,1,0)^{\top} \in \mathcal{A}_{\tau}^{k}$ for all $k$. We observe for all modifications a superlinear convergence. Comparing the behavior of the factor $\alpha_{p, l}^{k-1}$ the first and the second modification show almost the same behavior. Although the factor $\alpha_{p, l}^{k-1}$ tends faster towards one for the third modification, 
TABle 2. Comparison between the exact $(m=" \infty ")$ and the inexact $(m=1)$ strategy with $c_{n}=c_{\tau}=100$ using (3.11). Tolerance for Ipdas strategy: $\varepsilon_{u}=10^{-10}$.

\begin{tabular}{c|c||c|c||c|c||c|c}
\hline \multicolumn{2}{c||}{ strategy } & \multicolumn{2}{c||}{ exact } & \multicolumn{2}{c||}{ inexact } & \multicolumn{2}{c}{ nested } \\
\hline level $l$ & DOF & $K_{l}$ & MG-steps & $M_{l}$ & MG-steps & $M_{l}$ & MG-steps \\
\hline \hline 1 & 27 & 3 & 41 & 3 & 11 & 2 & 10 \\
\hline 2 & 125 & 3 & 44 & 3 & 13 & 2 & 12 \\
\hline 3 & 729 & 4 & 59 & 4 & 14 & 5 & 13 \\
\hline 4 & 4913 & 6 & 65 & 6 & 14 & 7 & 12 \\
\hline 5 & 35937 & 7 & 85 & 8 & 17 & 7 & 14 \\
\hline \hline
\end{tabular}

we observe a slower convergence. For the factor $\beta_{p, l}^{k-1}$ we observe a better behavior for the first modification than for the second one. From now on, we use modification one for all computations.

Exact versus inexact method. We next compare the exact version, i.e., $m=$ " $\infty$ ", of Algorithm 1 with the inexact version. In the inexact version, we use $m=1$, i.e., we update the active and inactive sets after each multigrid step. We denote by $K_{l}$ the iteration step in which the correct active and inactive sets are found for the first time and never change later in the iteration. For the inexact approach, we denote this step by $M_{l}$. Table 2 shows the numbers $K_{l}$ and $M_{l}$ on each level and the necessary numbers of multigrid iterations to solve the full nonlinear problem on level $l$. We observe that the numbers $K_{l}$ and $M_{l}$ are almost the same. They seem to depend linearly on the level $l$. Therefore, there is no need to solve the linear system exactly. Furthermore, we compare the inexact approach, where we start with $\boldsymbol{u}_{h}^{0,0}=\mathbf{0}$ and $\boldsymbol{\lambda}_{h}^{0}=\mathbf{0}$ on each level, with the nested approach in which we inherit $\boldsymbol{u}_{h}^{0,0}$ and $\boldsymbol{\lambda}_{h}^{0}$ on level $l+1$ from level $l$. The values $K_{l}$ and the necessary numbers of multigrid iteration steps are shown in the last column of Table 2. We note that the inexact primal-dual active set strategy can be interpreted as a non-linear multigrid method.

Influence of the parameter $c_{\tau}$. In a last test, we investigate the influence of the parameter $c_{\tau}$ on our algorithm. Recall that $c_{\tau}$ can be seen as weight for the tangential distortion $\boldsymbol{u}_{\tau, p}$ in the sum with the tangential component $\boldsymbol{\lambda}_{\tau, p, s}$ of the Lagrange multiplier. Thus, it plays a similar role for the tangential component as $c_{n}$ for the normal component. In Table 3, we compare the numbers $K_{l}$ and the numbers MG of necessary multigrid steps for different values of $c_{\tau}$, where we fix $c_{n}=100$ and use the inexact approach $m=1$. As 
TABlE 3. Comparison between different values for $c_{\tau}$ for the inexact strategy $(m=1)$ using $c_{n}=100$ and $\varepsilon_{u}=10^{-10}$. Initialization: $\boldsymbol{u}_{b}^{0,0}=\mathbf{0}, \boldsymbol{\lambda}_{h}^{0}=\mathbf{0}$ for all levels $l$.

\begin{tabular}{c||c|c||c|c||c|c||c|c||c|c}
\hline \hline \multicolumn{1}{c||}{$c_{\tau}$} & \multicolumn{2}{c||}{1} & \multicolumn{2}{c||}{10} & \multicolumn{2}{c||}{100} & \multicolumn{2}{c||}{1000} & \multicolumn{2}{c}{10000} \\
\hline level $l$ & $K_{l}$ & $\mathrm{MG}$ & $K_{l}$ & $\mathrm{MG}$ & $K_{l}$ & $\mathrm{MG}$ & $M_{l}$ & $\mathrm{MG}$ & $M_{l}$ & $\mathrm{MG}$ \\
\hline \hline 1 & 3 & 12 & 3 & 11 & 3 & 11 & 3 & 11 & 3 & 11 \\
\hline 2 & 3 & 12 & 3 & 12 & 3 & 13 & 3 & 13 & 4 & 13 \\
\hline 3 & 4 & 14 & 4 & 14 & 4 & 14 & 4 & 14 & 4 & 14 \\
\hline 4 & 6 & 15 & 6 & 14 & 6 & 14 & 6 & 14 & 6 & 14 \\
\hline 5 & - & - & 8 & 17 & 8 & 17 & 9 & 17 & 9 & 17 \\
\hline \hline
\end{tabular}

can be seen, the algorithm behaves quite stable and independent of $c_{\tau}$, if $c_{\tau}$ is large enough. For this example, we find $c_{\tau} \geq 10$. For $c_{\tau}=1$ the algorithm does not converge on Level 5. A very similar behavior is observed in [HW05] with respect to the parameter $c_{n}$. Thus, in general it appears advantageous to choose both $c_{\tau}$ and $c_{n}$ in order to balance the different scales of the distortion $\boldsymbol{u}_{h}$ and the Lagrange multiplier $\boldsymbol{\lambda}_{h}$.

\section{Fixpoint algorithm and numerical examples for Coulomb friction}

In this section, we extend Algorithm 1 to contact problems with Coulomb friction and give a numerical example showing the performance of the method. For Coulomb friction, the friction bound $g_{p}=\mathfrak{F}\left|\lambda_{n, p, s}\right|$ needs to be iteratively adjusted using the normal component of the Lagrange multiplier. Therefore, we get a further outer loop for the update of the friction bound.

\section{Algorithm 2: Ipdas fixed point strategy for contact with 3D Coulomb friction}

(0) Set $k=1$ and choose $c_{n}>0, c_{\tau}>0, m \in \mathbb{N}$ and $k_{f} \in \mathbb{N}$.

Initialize $\boldsymbol{u}_{h}^{0,0}$ and $\boldsymbol{\lambda}_{h}^{0}$.

(1) If $\bmod _{k_{f}}(k-1)=0$, set $k_{c}=k-1$ and update the friction bound by

$$
g_{p}^{k_{c}}=\mathfrak{F} \max \left\{0, \lambda_{n, p, s}^{k_{c}}\right\}, \quad p \in \mathcal{S}
$$


(2) Define the active and inactive sets by

$$
\begin{aligned}
\mathcal{A}_{n}^{k} & :=\left\{p \in \mathcal{S}: \lambda_{n, p, s}^{k-1}+c_{n}\left(u_{n, p}^{k-1, m}-d_{p}\right)>0\right\}, \\
\mathcal{I}_{n}^{k} & :=\left\{p \in \mathcal{S}: \lambda_{n, p, s}^{k-1}+c_{n}\left(u_{n, p}^{k-1, m}-d_{p}\right) \leq 0\right\}, \\
\mathcal{A}_{\tau}^{k} & :=\left\{p \in \mathcal{S}:\left\|\boldsymbol{\lambda}_{\tau, p, s}^{k-1}+c_{\tau} \boldsymbol{u}_{\tau, p}^{k-1, m}\right\|-g_{p}^{k_{c}}>0\right\}, \\
\mathcal{I}_{\tau}^{k} & :=\left\{p \in \mathcal{S}:\left\|\boldsymbol{\lambda}_{\tau, p, s}^{k}+c_{\tau} \boldsymbol{u}_{\tau, p}^{k-1, m}\right\|-g_{p}^{k_{c}} \leq 0\right\} .
\end{aligned}
$$

(3) For $i=1, \ldots, m$, compute

$$
\boldsymbol{u}_{h}^{k, i}=M G\left(\boldsymbol{u}_{h}^{k, i-1}, \mathcal{A}_{n}^{k}, \mathcal{I}_{n}^{k}, \mathcal{A}_{\tau}^{k}, \mathcal{I}_{\tau}^{k}, \boldsymbol{u}_{h}^{k-1, m}, \boldsymbol{\lambda}_{h}^{k-1}\right) .
$$

(4) Compute the Lagrange multiplier as

$$
\boldsymbol{\lambda}_{h}^{k}=D^{-1}\left(\boldsymbol{f}_{\mathcal{S}}-A_{\mathcal{S} \star} \boldsymbol{u}_{h}^{k, m}\right) .
$$

(5) If $k>k_{f}$ and $\left\|\boldsymbol{u}_{h}^{k, m}-\boldsymbol{u}_{h}^{k_{c}, m}\right\| /\left\|\boldsymbol{u}_{h}^{k, m}\right\|<\varepsilon_{u}$ stop.

(6) Set $\boldsymbol{u}_{h}^{k+1,0}=\boldsymbol{u}_{h}^{k, m}$ and $k=k+1$ and go to step (1).

In this algorithm, we denote by $\bmod _{*} *$ the modula-operator. Comparing this algorithm with Algorithm 1 for Tresca friction, we remark that here we update the friction bound after $k_{f}$ steps of the (inexact) active set strategy. This update is done in step (1). Since we do not solve the resulting linear problems exactly, it is not guaranteed that $\lambda_{n, p, s}^{k} \geq 0$ for all $p \in \mathcal{S}$. Therefore, we set $g_{p}=\mathfrak{F} \max \left\{0, \lambda_{n, p, s}^{k}\right\}$. For the choice $m=k_{f}=1$, the friction bound and the active and inactive sets are updated after each multigrid step. As stopping criterion, we use the relative error between the actual solution $\boldsymbol{u}_{h}^{k, m}$ and the solution for the last friction bound $\boldsymbol{u}_{h}^{k_{c}, m}$. For the choice $m=" \infty "$ and $k_{f}=" \infty$ ", we get the exact version of the algorithm. In this case, we solve the resulting Tresca friction problem exactly for each friction bound. Obviously, this approach is rather costly. However, for a small friction coefficient $\mathfrak{F}$ it can be shown that this discrete fixed point mapping is contractive and thus converges; see [NJH80].

5.1. Example. In this section, we study the performance of Algorithm 2 for Coulomb friction. This friction model is physically more realistic than the Tresca model, since only points that are in contact with the obstacle are points for which friction occurs. Points on the contact boundary with a 
positive distance to the obstacle are traction-free, i.e., we apply homogeneous Neumann conditions.
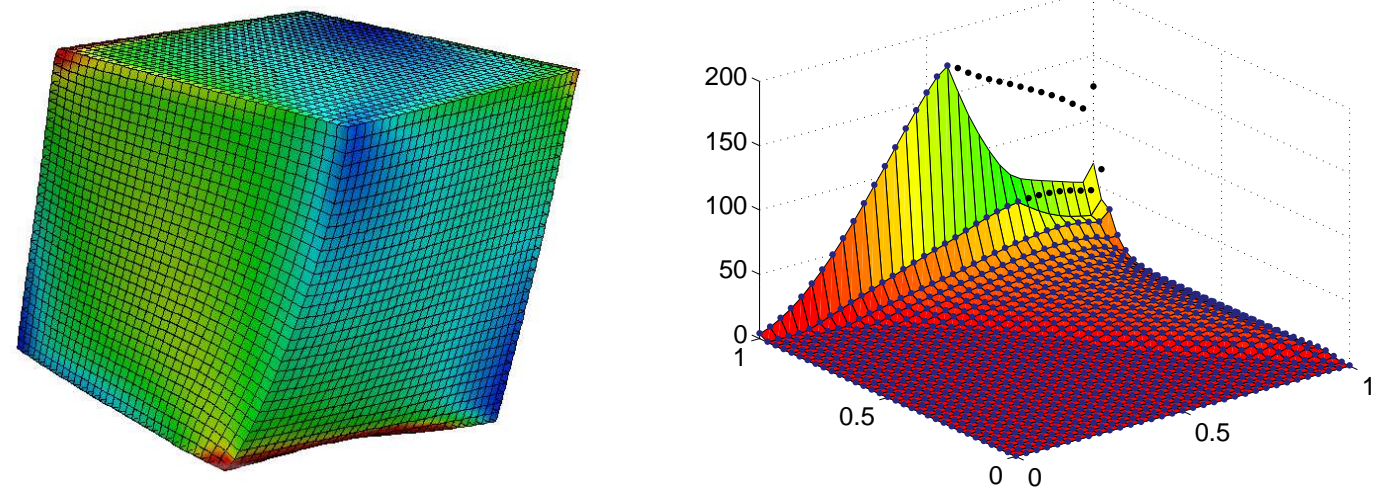

Figure 4. Distorted body with effective von Mises stress $\sigma_{\text {eff }}$ (left); the lower surface in the plot is subject to contact and Coulomb friction. Visualization of the friction bound $\mathfrak{F}\left|\lambda_{n, p}\right|$ (small balls) and of $\left\|\boldsymbol{\lambda}_{\tau, p}\right\|$ (right).
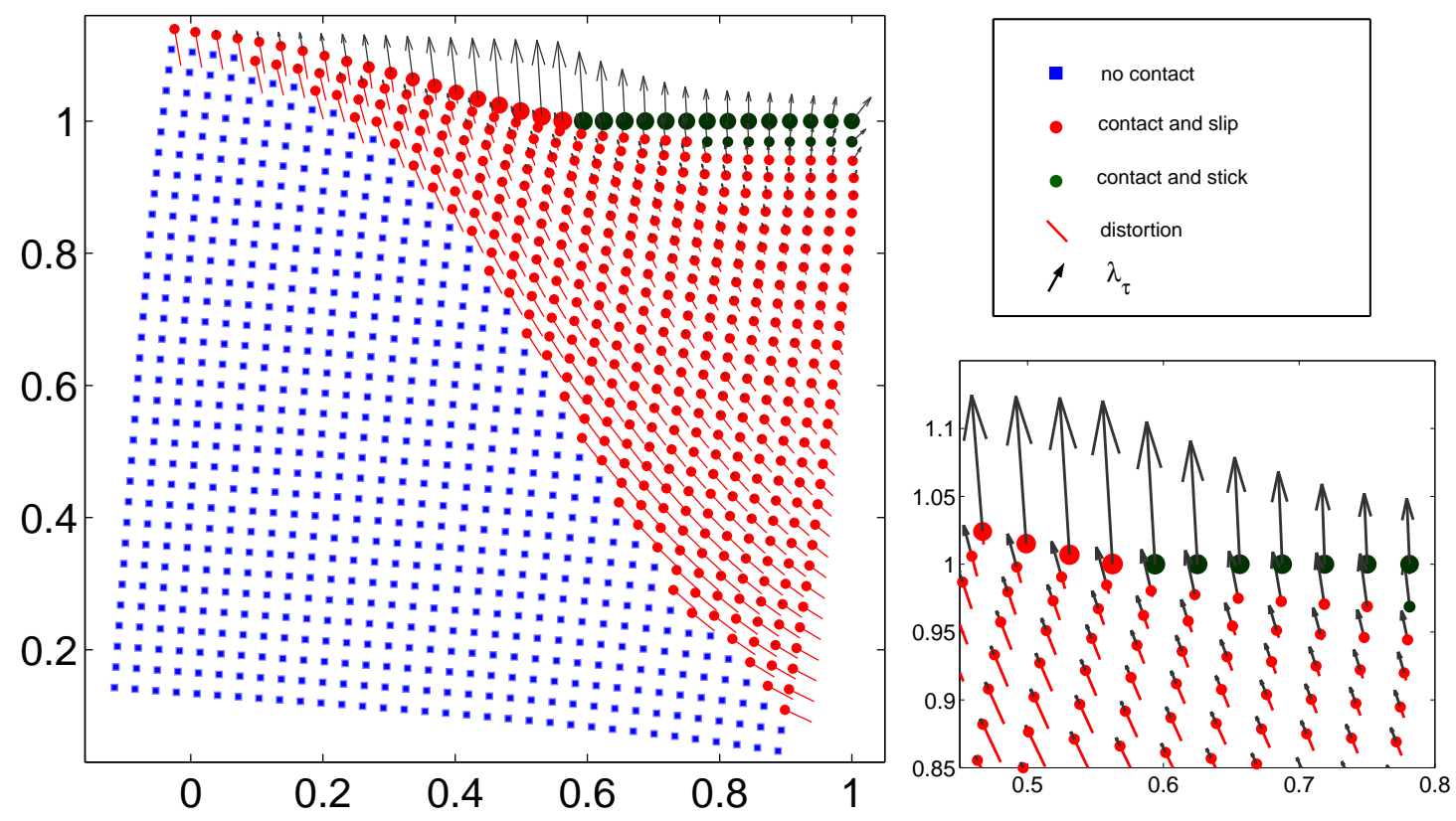

Figure 5. Visualization of the solution at the nodes $p \in \mathcal{S}$ for Coulomb friction on level 5 (left), explaining legend (upper right) and cutout (lower right).

Recall that in the Coulomb law, the friction bound becomes $g_{p}=\mathfrak{F}\left|\lambda_{n, p, s}\right|$ and thus depends on the actual distortion. In the following tests, we consider 
the same geometry and data as for the example of Section 4.1 and choose the friction coefficient $\mathfrak{F} \equiv 1$. The distorted cube can be seen in the left plot of Figure 4. Note that its distortion is significantly different from the one obtained with the Tresca law; (Figure 1). In the right of Figure 4, we visualize the constraint $\left\|\boldsymbol{\lambda}_{\tau, p, s}\right\| \leq \mathfrak{F}\left|\lambda_{n, p, s}\right|$ that holds for all $p \in \mathcal{S}$. The few nodes where this inequality holds in a strict sense are nodes in contact with the obstacle that, at the same time, stick to this obstacle, i.e., $u_{n, p}=d_{p}=0$ and $\boldsymbol{u}_{\tau, p}=\mathbf{0}$. We remark that the solution has a singularity at the node $(1,1,0)^{\top}$.

The Coulomb friction law. As for Tresca friction, (Figure 2), we also visualize the different types of contact nodes for the Coulomb law. Note that, in contrast to the Tresca model, the Coulomb law only allows three types of nodes, since nodes that are not in contact with the obstacle are not subject to any (friction) constraints. The size of the nodes in Figure 5 is proportional to the normal contact force $\left|\lambda_{n, p}\right|$.
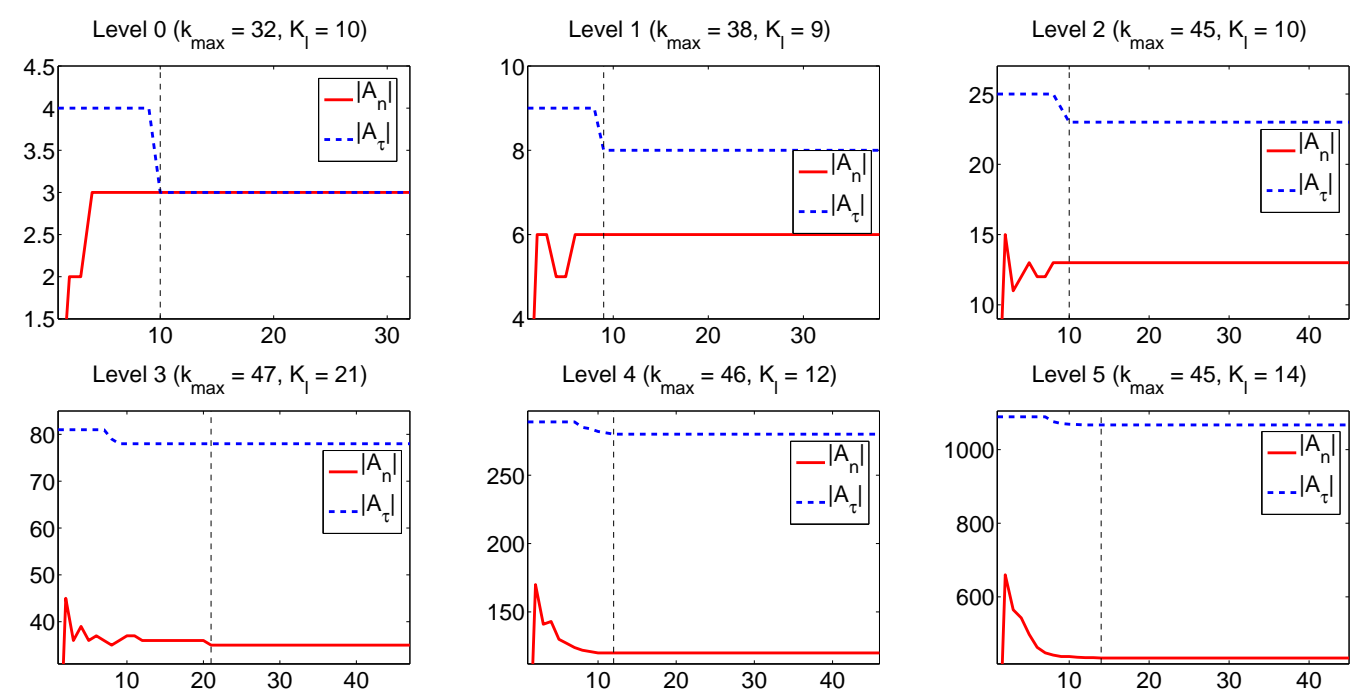

Figure 6. Behavior of Algorithm 2: Numbers $\left|\mathcal{A}_{n}^{k}\right|$ and $\left|\mathcal{A}_{\tau}^{k}\right|$ of active nodes in each iteration step $k$ on each level $l$. We used the parameters $c_{n}=c_{\tau}=100, m=k_{f}=1$ and $\varepsilon_{u}=10^{-10}$. Initialization: $\boldsymbol{u}_{h}^{0,0}=\mathbf{0}$ and $\boldsymbol{\lambda}_{h}^{0}=\mathbf{0}$.

Performance of the algorithm. We apply the inexact version of Algorithm 2 with $m=k_{f}=1$ to solve the contact problem with Coulomb friction. We use $c_{n}=c_{\tau}=100, \varepsilon_{u}=10^{-10}$ and initialize the iteration with $\boldsymbol{u}_{h}^{0,0}=\mathbf{0}$ and $\boldsymbol{\lambda}_{h}^{0}=\mathbf{0}$ on each level. Figure 6 shows the behavior of our algorithm on various 
TABLE 4. Behavior of Algorithm 2: Necessary numbers $k_{\max }$ and $K_{l}$ on each level $l$ for $c_{n}=c_{\tau}=100, m=k_{f}=1$ and $\varepsilon_{u}=10^{-10}$.

\begin{tabular}{c|c||c|c||c|c}
\hline \hline \multirow{2}{*}{ level } & \multirow{2}{*}{$l$} & \multicolumn{1}{|c||}{$\mathrm{DOF}$} & \multicolumn{2}{c||}{$\boldsymbol{u}_{h}^{0,0}=\mathbf{0}, \boldsymbol{\lambda}_{h}^{0}=\mathbf{0}$} & \multicolumn{2}{c}{ nested approach } \\
\cline { 3 - 6 } & & $K_{l}$ & $k_{\max }$ & $K_{l}$ & $k_{\max }$ \\
\hline \hline 0 & 8 & 10 & 32 & 10 & 32 \\
\hline 1 & 27 & 9 & 38 & 3 & 34 \\
\hline 2 & 125 & 10 & 45 & 5 & 32 \\
\hline 3 & 729 & 21 & 47 & 3 & 37 \\
\hline 4 & 4913 & 12 & 46 & 4 & 38 \\
\hline 5 & 35937 & 14 & 45 & 5 & 36 \\
\hline \hline
\end{tabular}

levels. The number of nodes contained in the active sets are plotted over the iteration steps $k$. We denote by $k_{\max }$ the necessary number of iteration steps and by $K_{l}$ the iteration step in which the correct active sets are found for the first time and do not change afterwards. The number $K_{l}$ is marked by a dashed vertical line in Figure 6 . We observe that both $k_{\max }$ and $K_{l}$ appear to be quite independent of the level $l$. Looking at Figure 6 more closely, we note that on each level there are only minor changes of the active nodes after $k=10$. Table 4 shows a comparison between the initialization $\boldsymbol{u}_{h}^{0,0}=\mathbf{0}$ and $\boldsymbol{\lambda}_{h}^{0}=\mathbf{0}$ on each level and the nested approach. As expected, the correct active sets are found earlier, and as a result fewer iterations are required for the nested approach.

\section{Numerical examples for two-body contact with Coulomb friction}

Now, we consider a curved contact interface subjected to Coulomb friction. A two-dimensional cross section of our geometry is shown in Figure 7. The lower domain $\Omega^{m}$, assumed to be the master side, models a spherical shell that is fixed at the outer boundary. Against this shell, we press the body modeled by the domain $\Omega^{s}$, which is assumed to be the slave side. At the top surface of $\Omega^{s}$, we apply the surface traction $\left(0,0,-150 \exp \left(-100 r^{2}\right)\right)^{\top}$, where $r$ denotes the distance to the midpoint of the top surface of $\Omega^{s}$. The geometry is given by $r_{i}=0.7, r_{a}=1.0, r=0.6, h=0.5$ and $d=0.3$. In $\Omega^{s}$, we use a Young modulus $E^{s}=300$ and a Poisson ratio $\nu^{s}=0.3$, while in $\Omega^{m}$ we have $E^{m}=400$ and $\nu^{m}=0.3$.

Discussion of the results $\&$ comparison for various friction coefficients. The results for the friction coefficient $\mathfrak{F}=0.7$ are shown in Figures 7-9. 

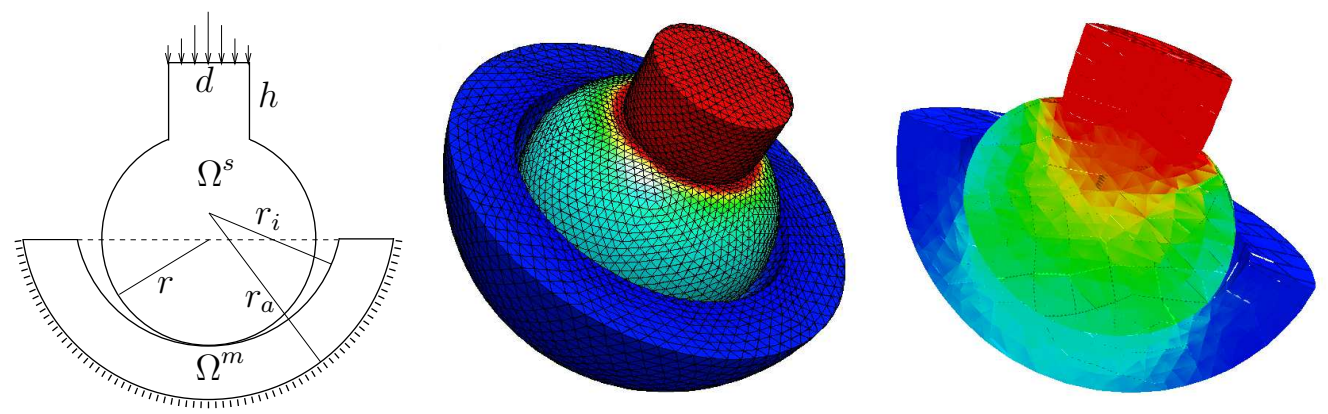

Figure 7. Problem definition (left), deformed mesh with effective von Mises stress $\sigma_{\text {eff }}$ on Level 3 (middle), two-dimensional cross section of the deformed mesh on Level 2 (right) for $\mathfrak{F}=0.7$.
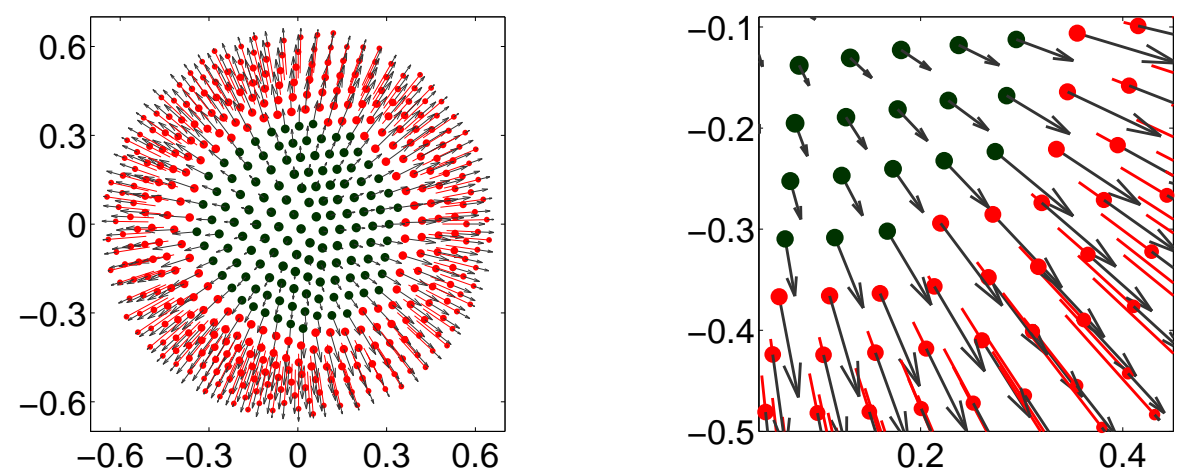

FiguRE 8. Visualization of the nodes being in contact together with the deformation and stress vectors on Level 3 for $\mathfrak{F}=0.7$ (left), and a cutout (right); for the legend we refer to Figure 5.
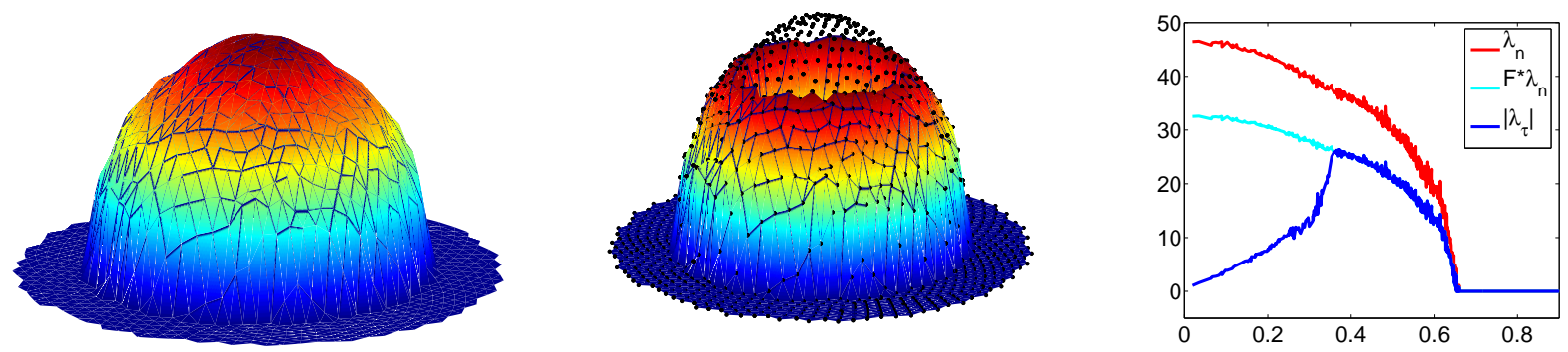

Figure 9. Visualization of $\lambda_{n}$ (left) and $\left\|\boldsymbol{\lambda}_{\tau}\right\|$ (middle) with the friction bound $0.7\left|\lambda_{n}\right|$ (dotted), and two-dimensional visualization of the Lagrange multipliers (right) on Level 3.

Figure 7 shows the deformed body with the effective von Mises stress $\sigma_{\text {eff }}$ on Level 3 and a two-dimensional cross section on Level 2. In Figure 8, we 

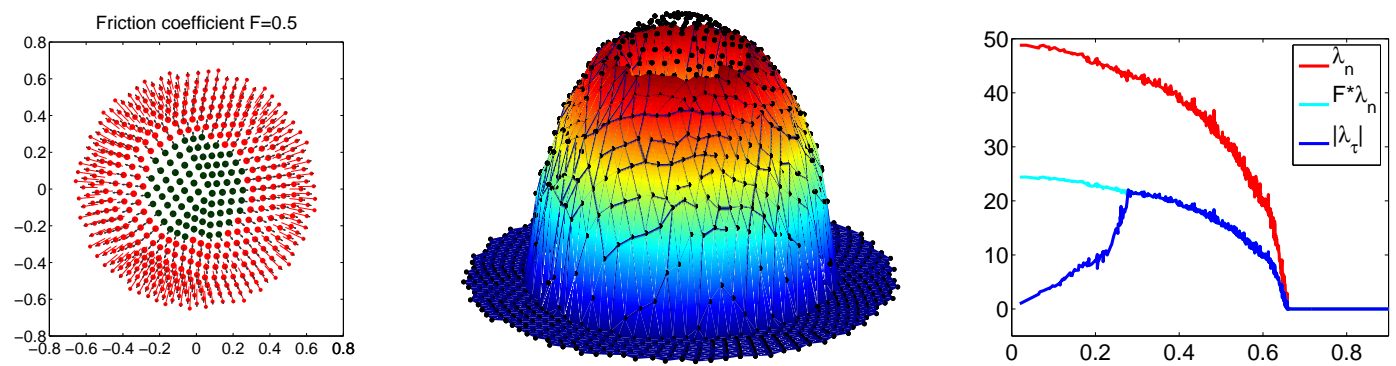

FiguRE 10. Visualization of the nodes being in contact, together with the deformation and the stress vectors (left), visualization of $\left\|\boldsymbol{\lambda}_{\tau}\right\|$ (middle) with the friction bound $0.5\left|\lambda_{n}\right|$ (dotted) and a two-dimensional visualization of the Lagrange multipliers (right).

show the nodes being in contact, their relative tangential slip $\left[\boldsymbol{u}_{\tau, p}\right]$ (lines) and the tangential contact pressure $\boldsymbol{\lambda}_{\tau, p}$ (arrows). We remark that the nodes in the middle with only an arrow are the sticky nodes, the others are slippy. Figure 9 shows on the left the normal part of the contact pressure $\lambda_{n}$ and in the middle the norm of the tangential part $\left\|\boldsymbol{\lambda}_{\tau}\right\|$. The dots represent the friction bound $0.7\left|\lambda_{n}\right|$. The two-dimensional plot on the right shows the normal and tangential part of the Lagrange multiplier and the friction bound for all nodes $p \in \mathcal{S}$ over their distance to the midpoint of the contact zone on the surface of $\Omega^{s}$. The small oscillations in the plot occur due to the fact that all nodes $p \in \mathcal{S}$ over the whole contact zone are presented and that we work with an unstructured mesh. The results for the same problem but with the smaller friction coefficient $\mathfrak{F}=0.5$ are shown in Figure 10. The number of sticky nodes decreases for smaller $\mathfrak{F}$.
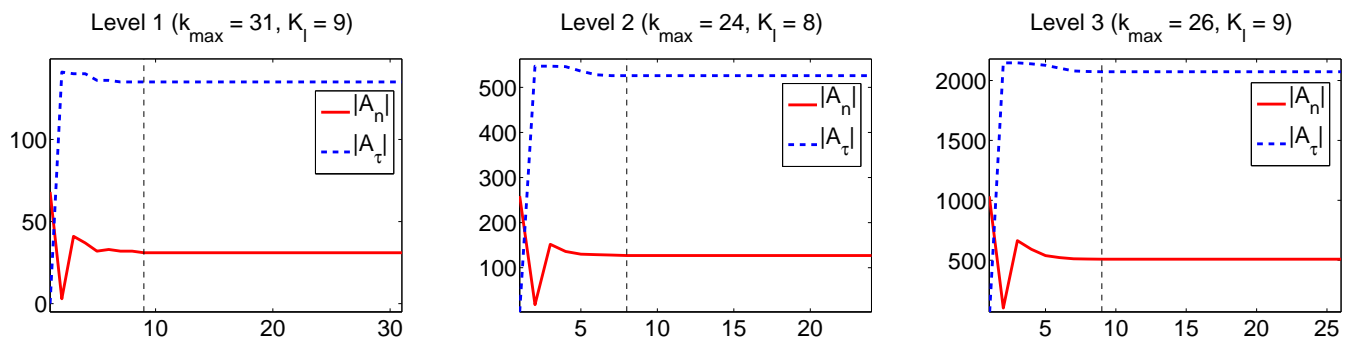

Figure 11. Behavior of Algorithm 2: Numbers $\left|\mathcal{A}_{n}^{k}\right|$ and $\left|\mathcal{A}_{\tau}^{k}\right|$ in each iteration step $k$ on Levels $1,2,3$ for $\mathfrak{F}=0.7$. We use the parameters $c_{n}=c_{\tau}=100, m=k_{f}=1$ and $\varepsilon_{u}=10^{-9}$. 
TABLE 5. Behavior of Algorithm 2: Necessary numbers $k_{\max }$ and $K_{l}$ on each level for $c_{n}=c_{\tau}=100, m=k_{f}=1, \varepsilon_{u}=10^{-9}$ and $\mathfrak{F} \equiv 0.7$.

\begin{tabular}{c|c||c|c||c|c}
\hline \hline \multirow{2}{*}{ leve } & \multirow{2}{*}{ DOF } & \multicolumn{2}{c||}{$\boldsymbol{u}_{p}^{0,0}=0.1 \boldsymbol{n}_{p}, \boldsymbol{\lambda}_{p}^{0}=0.0001 \boldsymbol{n}_{p}$} & \multicolumn{2}{c}{ nested approach } \\
\cline { 3 - 6 } & & $K_{l}$ & $k_{\max }$ & $K_{l}$ & $k_{\max }$ \\
\hline \hline 0 & 104 & 5 & 26 & 5 & 26 \\
\hline 1 & 541 & 9 & 31 & 5 & 23 \\
\hline 2 & 3384 & 8 & 24 & 6 & 21 \\
\hline 3 & 23694 & 9 & 26 & 5 & 20 \\
\hline \hline
\end{tabular}

Performance of the algorithm. We consider the performance of Algorithm 2 for the friction coefficient $\mathfrak{F}=0.7$. For the initialization we set $\boldsymbol{u}_{p}^{0,0}=0.1 \boldsymbol{n}_{p}$ for $p \in \mathcal{S}, \boldsymbol{u}_{p}^{0,0}=\mathbf{0}$ for $p \notin \mathcal{S}$ and $\boldsymbol{\lambda}_{p}^{0}=0.0001 \boldsymbol{n}_{p}$ on each level. Using the parameters $c_{n}=c_{\tau}=100, m=k_{f}=1$ and $\varepsilon_{u}=10^{-9}$, we get the performance of Algorithm 2 shown in Figure 11. Here, the number of nodes in $\left|\mathcal{A}_{n}^{k}\right|$ and $\left|\mathcal{A}_{\tau}^{k}\right|$ are shown for Level 1-3. Again, the dashed vertical line marks the step $K_{l}$ in which the correct active sets are found for the first time and remain unchanged. A comparison between this approach and the nested approach is shown in Table 5. The results obtained show qualitatively the same behavior as for the cube; see Section 5.1.
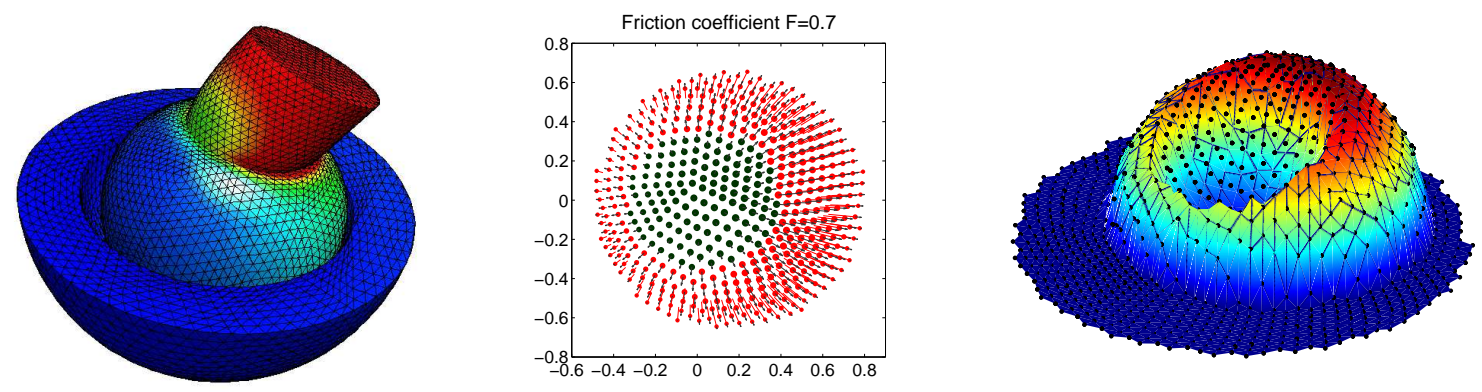

FiguRE 12. Nonsymmetric boundary data: Deformed mesh with effective von Mises stress $\sigma_{\text {eff }}$ on Level 3 (left), visualization of the nodes being in contact together with the deformation and the stress vectors (middle) and of $\left\|\boldsymbol{\lambda}_{\tau}\right\|$ (right) with the friction bound $0.7\left|\lambda_{n}\right|$ (dotted).

Problem with nonsymmetric boundary traction. For the next example, we use the same data and geometry as above (see Figure 7), but use a nonsymmetric boundary traction, namely, we apply the surface traction 
$\left(15,0,-150 \exp \left(-100 r^{2}\right)\right)^{\top}$ at the top surface. The results for $\mathfrak{F}=0.7$ are presented in Figure 12.

\section{Full Newton approach for multibody contact with Coulomb friction}

While our algorithm in Section 5 for the solution of the Coulomb friction problem is based on fixed point ideas, we now present a full Newton approach for the Coulomb frictional contact problem. The main advantage of this approach is its fast convergence which is due to the fact that the friction bound is updated in the Newton iteration and not via a fixed point loop. In this section, we apply a fast direct solver [SGF00] to solve the linear system in each Newton step. To derive the full Newton iteration, one replaces $g_{p}$ in (3.1) either by $g_{p}\left(u_{n, p}, \lambda_{n, p, s}\right):=\mathfrak{F} \max \left(0, \lambda_{n, p, s}\right)$ (see also [CKPS98,CP99]) or by $g_{p}\left(u_{n, p}, \lambda_{n, p, s}\right):=\mathfrak{F} \max \left(0, \lambda_{n, p, s}+c_{n}\left(u_{n, p}-d_{p}\right)\right)$ before deriving the Newton iteration step. The equivalence of these two choices follows from (4.1). In the sequel, we use the latter replacement since then the Newton-type iteration automatically takes the form of an active set method that, in each iteration estimates the three relevant sets for Coulomb friction (no contact, contact and stick, contact and slip). The resulting nonlinear complementarity function is

$$
\begin{aligned}
\mathcal{D}\left(\boldsymbol{u}_{h}, \boldsymbol{\lambda}_{h}\right):= & \max \left(\mathfrak{F}\left(\lambda_{n, p, s}+c_{n}\left(u_{n, p}-d_{p}\right)\right),\left\|\boldsymbol{\lambda}_{\tau, p, s}+c_{\tau} \boldsymbol{u}_{\tau, p}\right\|\right) \boldsymbol{\lambda}_{\tau, p, s} \\
& -\mathfrak{F} \max \left(0, \lambda_{n, p, s}+c_{n}\left(u_{n, p}-d_{p}\right)\right)\left(\boldsymbol{\lambda}_{\tau, p, s}+c_{\tau} \boldsymbol{u}_{\tau, p}\right) .
\end{aligned}
$$

Similarly as in Section 3, we derive a semismooth Newton step for the solution of $\mathcal{D}\left(\boldsymbol{u}_{h}, \boldsymbol{\lambda}_{h}\right)=0$ and (4.1). Using the notation $g_{p}^{k-1}:=\mathfrak{F}\left(\lambda_{n, p, s}^{k-1}+\right.$ $\left.c_{n}\left(u_{n, p}^{k-1}-d_{p}\right)\right)$, we obtain the following settings.

- On $\mathcal{I}_{n}^{k}:=\left\{p \in \mathcal{S}: g_{p}^{k-1} \leq 0\right\}$ (estimation for set of nodes not in contact):

$$
\lambda_{n, p, s}^{k}=0 \quad \text { and } \quad \lambda_{\tau, p, s}^{k}=0 .
$$

Note that $\mathcal{I}_{n}^{k} \subset \mathcal{A}_{\tau}^{k}$, where $\mathcal{A}_{\tau}^{k}:=\left\{p \in \mathcal{S}:\left\|\boldsymbol{\lambda}_{\tau, p, s}^{k-1}+c_{\tau} \boldsymbol{u}_{\tau, p}^{k-1}\right\|-g_{p}^{k-1} \geq 0\right\}$. We remark that the setting for $\boldsymbol{\lambda}_{\tau, p, s}^{k}$ is derived directly from (7.1) for that case, namely from $\left\|\boldsymbol{\lambda}_{\tau, p, s}+c_{\tau} \boldsymbol{u}_{\tau, p}\right\| \boldsymbol{\lambda}_{\tau, p, s}=0$.

- On $\mathcal{I}_{\tau}^{k}:=\left\{p \in \mathcal{S}:\left\|\boldsymbol{\lambda}_{\tau, p, s}^{k-1}+c_{\tau} \boldsymbol{u}_{\tau, p}^{k-1}\right\|-g_{p}^{k-1}<0\right\}$ : (estimation for set of sticky nodes):

$$
u_{n, p}^{k}=d_{p} \quad \text { and } \quad \boldsymbol{u}_{\tau, p}^{k}+\left(\mathfrak{F} \boldsymbol{u}_{\tau, p}^{k-1} / g_{p}^{k-1}\right) \lambda_{n, p, s}^{k}=\boldsymbol{u}_{\tau, p}^{k-1} .
$$


Note that $\mathcal{I}_{\tau}^{k} \subset \mathcal{A}_{n}^{k}$, where $\mathcal{A}_{n}^{k}:=\left\{p \in \mathcal{S}: g_{p}^{k-1}>0\right\}$.

- On $\mathcal{A}_{m}^{k}:=\mathcal{A}_{\tau}^{k} \cap \mathcal{A}_{n}^{k}=\left\{p \in \mathcal{S}:\left\|\boldsymbol{\lambda}_{\tau, p, s}^{k-1}+c_{\tau} \boldsymbol{u}_{\tau, p}^{k-1}\right\| \geq g_{p}^{k-1}>0\right\}$ (estimation for set of slippy nodes):

$$
\begin{aligned}
u_{n, p}^{k} & =d_{p} \quad \text { and } \\
-\boldsymbol{\lambda}_{\tau, p, s}^{k}+L_{p, l}^{k-1} \boldsymbol{u}_{\tau, p}^{k}+\mathfrak{F} \boldsymbol{v}_{p, l}^{k-1} \lambda_{n, p, s}^{k} & =\boldsymbol{r}_{p, l}^{k-1}+g_{p}^{k-1} \boldsymbol{v}_{p, l}^{k-1},
\end{aligned}
$$

where $\boldsymbol{v}_{p}^{k-1}:=\left(I d_{2}-M_{p}^{k-1}\right)^{-1}\left(\boldsymbol{\lambda}_{\tau, p, s}^{k-1}+c_{\tau} \boldsymbol{u}_{\tau, p}^{k-1}\right) /\left\|\boldsymbol{\lambda}_{\tau, p, s}^{k-1}+c_{\tau} \boldsymbol{u}_{\tau, p}^{k-1}\right\| \in \mathbb{R}^{2}$ and $g_{p}^{k-1}$ is used for the friction bound $g_{p}$ in the matrices $M_{p}^{k-1}$ and $L_{p}^{k-1}$.

Note that $\mathcal{I}_{n}^{k}, \mathcal{I}_{\tau}^{k}$, and $\mathcal{A}_{m}^{k}$ represent a disjoint splitting of $\mathcal{S}$. Comparing (7.2) and (7.3) with (3.5a) and (3.5b), respectively, we observe that the main difference is the term involving $\lambda_{n, p, s}^{k}$ on the left hand side of (7.2) and (7.3). Again we apply the modifications stated in Subsection 3.2 and replace the matrices $M_{p}^{k-1}$ and $L_{p}^{k-1}$ by $\tilde{M}_{p, l}^{k-1}$ and $\tilde{L}_{p, l}^{k-1}$, respectively. Therefore, we use instead of $\boldsymbol{v}_{p}^{k-1}$ the vector $\tilde{\boldsymbol{v}}_{p, l}^{k-1}:=\left(I d_{2}-\beta_{p, l}^{k-1} \tilde{M}_{p, l}^{k-1}\right)^{-1}\left(\boldsymbol{\lambda}_{\tau, p, s}^{k-1}+c_{\tau} \boldsymbol{u}_{\tau, p}^{k-1}\right) /\left\|\boldsymbol{\lambda}_{\tau, p, s}^{k-1}+c_{\tau} \boldsymbol{u}_{\tau, p}^{k-1}\right\|$. Now, we briefly state the algebraic representation of the above system, where we use in addition to the notation introduced in Section 3

$$
G:=\operatorname{diag}\left\{g_{p}^{k-1} I d_{2}\right\}_{p \in \mathcal{S}} \in \mathbb{R}^{2|\mathcal{S}| \times 2|\mathcal{S}|} .
$$

and

$$
U:=\operatorname{diag}\left\{\boldsymbol{u}_{\tau, p}^{k-1}\right\}_{p \in \mathcal{S}}, \quad V:=\operatorname{diag}\left\{\tilde{\boldsymbol{v}}_{p, l}^{k-1}\right\}_{p \in \mathcal{S}} \in \mathbb{R}^{2|\mathcal{S}| \times|\mathcal{S}|} .
$$

We use a subblock notation for the matrices, e.g., $G_{\mathcal{I}_{\tau}^{n}} \ldots$ Defining $\tilde{\mathcal{N}}:=$ $\mathcal{N} \cup \mathcal{I}_{n}^{k}$, we obtain after eliminating the Lagrange multiplier, the following linear system to be solved in each full Newton step for the Coulomb problem as

$$
\left(\begin{array}{ccc}
A_{\tilde{\mathcal{N}} \tilde{\mathcal{N}}} & A_{\tilde{\mathcal{N}} \mathcal{I}_{\tau}^{k}} & A_{\tilde{\mathcal{N}} \mathcal{A}_{m}^{k}}^{k} \\
0 & N_{\mathcal{I}_{\tau}^{k}} & 0 \\
0 & 0 & N_{\mathcal{A}_{m n}^{k}} \\
K_{\mathcal{I}_{\tau}^{k}} A_{\mathcal{I}_{\tau}^{k} \tilde{\mathcal{N}}} & K_{\mathcal{I}_{\tau}^{k}} A_{\mathcal{I}_{\mathcal{T}}^{k} \mathcal{T}_{\tau}^{k}-G_{\mathcal{I}_{\tau}^{k}} T_{\mathcal{I}_{\tau}^{k}}} & K_{\mathcal{I}_{\tau}^{k}} A_{\mathcal{I}_{\tau}^{k} \mathcal{A}_{m}^{k}}^{k} \\
T_{\mathcal{A}_{m}^{k}}^{\prime} A_{\mathcal{A}_{m}^{k} \tilde{\mathcal{N}}} & T_{\mathcal{A}_{m}^{k}}^{\prime} A_{\mathcal{A}_{m}^{k} \mathcal{I}_{\tau}^{k}} & T_{\mathcal{A}_{m}^{k}}^{\prime} A_{\mathcal{A}_{m}^{k}}^{k} \mathcal{A}_{m}^{k}+L_{\mathcal{A}_{m}^{k}} T_{\mathcal{A}_{m}^{k}}^{k}
\end{array}\right)\left(\begin{array}{c}
\boldsymbol{u}_{\tilde{\tilde{N}}}^{k} \\
\boldsymbol{u}_{\mathcal{I}_{\tau}^{k}}^{k} \\
\boldsymbol{u}_{\mathcal{A}_{m}^{k}}^{k}
\end{array}\right)=\left(\begin{array}{c}
\boldsymbol{f}_{\tilde{\mathcal{N}}} \\
d_{\mathcal{I}_{\tau}^{k}} \\
d_{\mathcal{A}_{m}^{k}}^{k} \\
K_{\mathcal{I}_{\tau}^{k}} \boldsymbol{f}_{\mathcal{I}_{\tau}^{k}}^{k} \boldsymbol{j}_{\mathcal{I}_{\tau}^{k}} \\
T_{\mathcal{A}_{m}^{k}}^{\prime} \boldsymbol{f}_{\mathcal{A}_{m}^{k}}^{k}+\boldsymbol{r}_{\mathcal{A}_{m n}^{k}}^{\prime}
\end{array}\right)
$$

with the notation

$$
T_{\mathcal{A}_{m}^{k}}^{\prime}:=T_{\mathcal{A}_{m}^{k}}-\mathfrak{F} V_{\mathcal{A}_{m}^{k}} N_{\mathcal{A}_{m n}^{k}}, \quad K_{\mathcal{I}_{\tau}^{k}}:=\mathfrak{F} U_{\mathcal{I}_{\tau}^{k}} N_{\mathcal{I}_{\tau}^{k}}
$$


and

$$
\boldsymbol{r}_{\mathcal{A}_{m n}^{k}}^{\prime}:=\boldsymbol{r}_{\mathcal{A}_{m}^{k}}+G_{\mathcal{A}_{m}^{k}} V_{\mathcal{A}_{m}^{k}}, \quad \boldsymbol{j}_{\mathcal{I}_{\tau}^{k}}:=G_{\mathcal{I}_{\tau}^{k}} U_{\mathcal{I}_{\tau}^{k}} .
$$

We observe, comparing this system with (3.15), that here the normal and tangential components are pointwise coupled by the lines four and five.
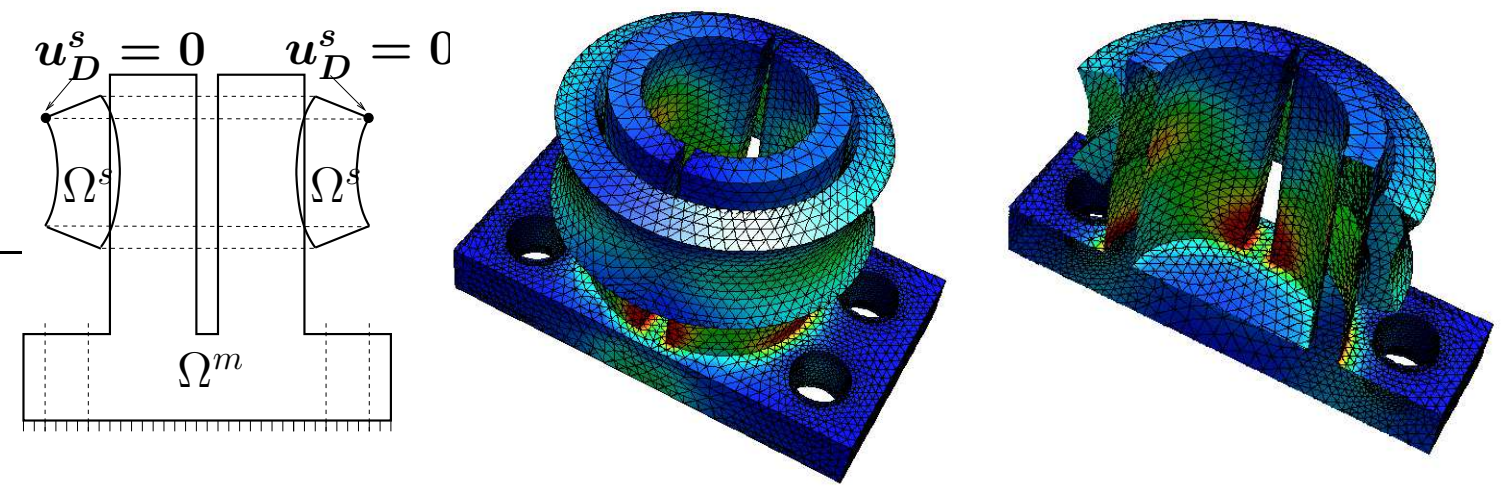

Figure 13. Problem definition (left), deformed mesh with effective von Mises stress $\sigma_{\text {eff }}$ (middle), two-dimensional cross section of the deformed mesh (right) for $\mathfrak{F}=0.7$.

Numerical Example. As example, we consider the situation presented in the left of Figure 13, where a two-dimensional cross section of the problem definition is shown. The ring is fixed on its upper outer edge and the tool on its bottom. Note that the bodies penetrate in their reference configuration. We use Young modulus $E^{m}=8.13 \times 10^{8}$ and a Poisson ratio $\nu^{m}=0.3$ for the inner tool modeled by $\Omega^{m}$, and $E^{s}=9 \times 10^{7}, \nu^{s}=0.3$ for the outer ring being the slave domain $\Omega^{s}$. The friction coefficient is $\mathfrak{F}=0.7$. The deformed mesh with effective von Mises stress $\sigma_{\text {eff }}$ is shown in the middle and the right of Figure 13. Figure 14 shows the possible contact nodes on the ring being the slave side. The nodes without a line are nodes not being in contact with the inner tool.

Comparison between fixpoint Newton and full Newton approach. To show the behavior of the full Newton approach, we compare the convergence rates of the Lagrange multiplier with those obtained from the fixpoint Newton approach given by Algorithm 2 with $m=" \infty "$ and $k_{f}=1$. Our algorithm is initialized with $\boldsymbol{u}_{h}^{0,0}=\mathbf{0}$ and $\boldsymbol{\lambda}_{h}^{0}=\mathbf{0}$, and the parameters $c_{n}=c_{\tau}=10^{8}$ and the tolerance $\varepsilon_{u}=10^{-9}$ are used. The finite element mesh consists of 66.600 degrees of freedom. In the left of Figure 15, the relative error $\left\|\boldsymbol{\lambda}_{h}^{k}-\boldsymbol{\lambda}_{h}^{*}\right\| /\left\|\boldsymbol{\lambda}_{h}^{*}\right\|$ is shown for the fixed point and the full Newton approach 

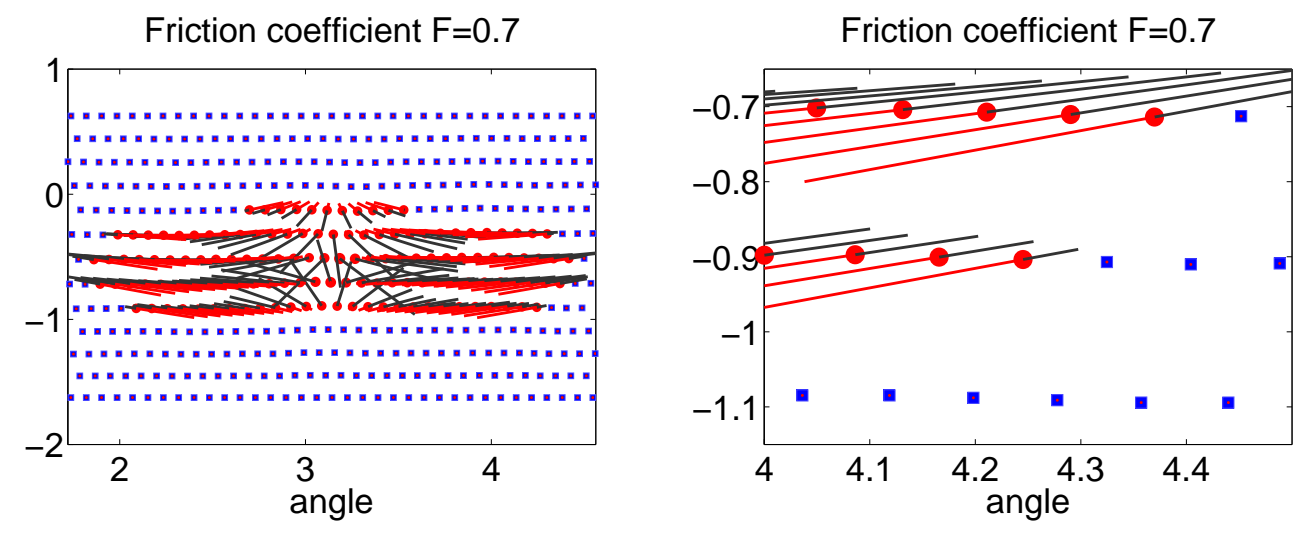

FiguRE 14. Visualization of the nodes being in contact, together with the deformation and stress vectors for $\mathfrak{F}=0.7$ (left), and a cutout (right); for the legend we refer to Figure 5.
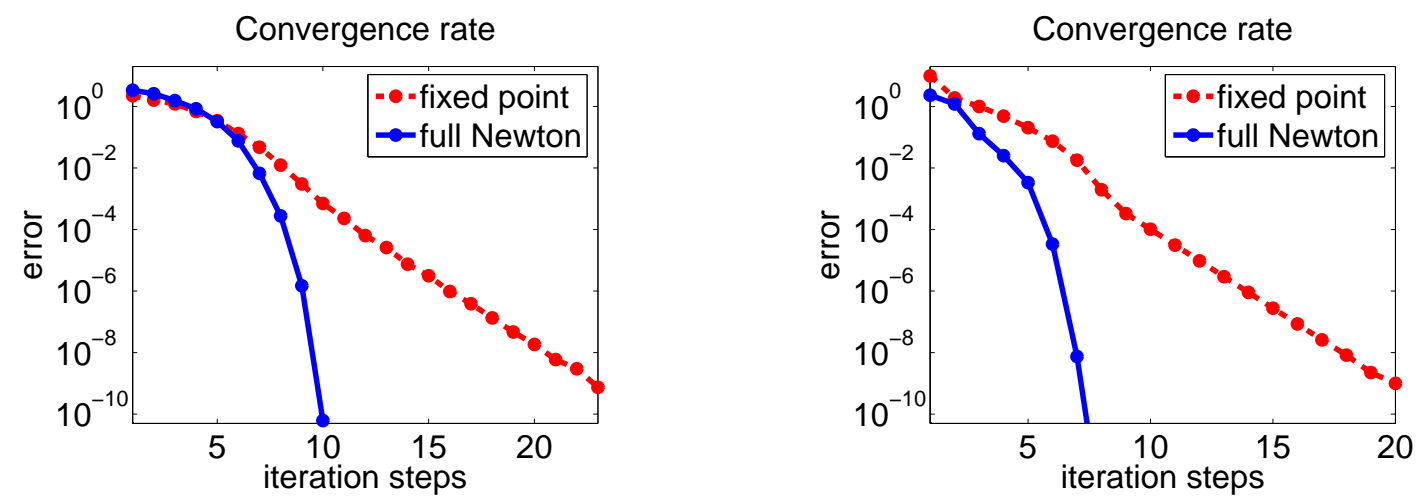

Figure 15. Convergence rates for the examples of Figure 13 (left) and Figure 7 (right) for the fixpoint Newton and the full Newton approach.

in a logarithmic scale. We observe superlinear convergence of the full Newton approach, and only half of the iteration steps compared with the fixed point approach are required. For the fixed point approach the convergence rate $\left\|\boldsymbol{\lambda}_{h}^{k+1}-\boldsymbol{\lambda}_{h}^{*}\right\| /\left\|\boldsymbol{\lambda}_{h}^{k}-\boldsymbol{\lambda}_{h}^{*}\right\|$ tends to approximately 0.32 . In the right of Figure 15, we show the comparison between the two approaches for the example presented in Figure 7. Again we observe the same behavior as for the example before.

\section{References}

[AC91] P. Alart and A. Curnier. A mixed formulation for frictional contact problems prone to Newton like solution methods. Comp. Meth. Appl. Mech. Engng., 92:353-375, 1991. 
[ACCO00] K. Andersen, E. Christiansen, A. Conn, and M. Overton. An efficient primal-dual interior-point method for minimizing a sum of Euclidean norms. SIAM J. Sci. Comput., 22(1):243-262 (electronic), 2000.

[BB00] F. Ben Belgacem. Numerical simulation of some variational inequalities arisen from unilateral contact problems by the finite element methods. SIAM J. Numer. Anal., 37(4):1198-1216 (electronic), 2000.

$\left[\mathrm{BBJ}^{+} 97\right]$ P. Bastian, K. Birken, K. Johannsen, S. Lang, N. Neuß, H. Rentz-Reichert, and C. Wieners. UG - a flexible software toolbox for solving partial differential equations. Comput. Vis. Sci., 1:27-40, 1997.

[CGM99] T.F. Chan, G.H. Golub, and P. Mulet. A nonlinear primal-dual method for total variation-based image restoration. SIAM J. Sci. Comput., 20(6):1964-1977, 1999.

[CKPS98] P. Christensen, A. Klarbring, J. Pang, and N. Strömberg. Formulation and comparison of algorithms for frictional contact problems. Internat. J. Numer. Methods Engrg., 42(1):145-173, 1998.

[CP99] P. Christensen and J. Pang. Frictional contact algorithms based on semismooth Newton methods. In Reformulation: nonsmooth, piecewise smooth, semismooth and smoothing methods, volume 22 of Appl. Optim., pages 81-116. Kluwer Acad. Publ., Dordrecht, 1999.

[DHK $\left.{ }^{+} 05\right]$ Z. Dostál, D. Horák, R. Kučera, V. Vondrák, J. Haslinger, J. Dobiáš, and S. Pták. FETI based algorithms for contact problems: scalability, large displacements and 3D Coulomb friction. Comput. Methods Appl. Mech. Engrg., 194(2-5):395-409, 2005.

[EJK05] C. Eck, J. Jarusek, and M. Krbec. Unilateral contact problems. Variational methods and existence theorems. Chapman \& Hall/CRC, Boca Raton, Fl., 2005.

[FK06] K. Fackeldey and R. Krause. Solving frictional contact problems with multigrid efficiency. In Proceedings of the 16th International Conference on Domain Decomposition Methods 2005, New York, 2006.

[FW05] K. Fischer and P. Wriggers. Frictionless 2d Contact formulations for finite deformations based on the mortar method. Comput. Mech., 36:226-244, 2005.

[FW06] K. Fischer and P. Wriggers. Mortar based frictional contact formulation for higher order interpolations using the moving friction cone. Comput. Methods Appl. Mech. Engrg., 2006. to appear.

[HDK02] J. Haslinger, Z. Dostál, and R. Kučera. On a splitting type algorithm for the numerical realization of contact problems with Coulomb friction. Comput. Methods Appl. Mech. Engrg., 191(21-22):2261-2281, 2002.

[HHNL88] I. Hlaváček, J. Haslinger, J. Nečas, and J. Lovíšek. Solution of Variational Inequalities in Mechanics. Number 66 in Applied Mathematical Sciences. Springer, New York, 1988.

[HIK03] M. Hintermüller, K. Ito, and K. Kunisch. The primal-dual active set strategy as a semismooth Newton method. SIAM J. Optim., 13(3):865-888, 2003.

[HKD04] J. Haslinger, R. Kučera, and Z. Dostál. An algorithm for the numerical realization of 3D contact problems with Coulomb friction. J. Comput. Appl. Math., 164/165:387-408, 2004.

[HKK04] M. Hintermüller, V. Kovtunenko, and K. Kunisch. Semismooth Newton methods for a class of unilaterally constrained variational problems. Adv. Math. Sci. Appl., 14(2):513$535,2004$.

[HL02] P. Hild and P. Laborde. Quadratic finite element methods for unilateral contact problems. Appl. Numer. Math., 41:410-421, 2002.

[HMW06] S. Hüeber, A. Matei, and B. Wohlmuth. Efficient algorithms for problems with friction. SIAM Journal on Scientific Computing, 2006. To appear. 
[HS06] M. Hintermüller and G. Stadler. An infeasible primal-dual algorithm for total variationbased inf-convolution-type image restoration. SIAM J. Sci. Comput., 28(1):1-23, 2006.

[HW05] S. Hüeber and B. Wohlmuth. A primal-dual active set strategy for non-linear multibody contact problems. Computer Methods in Applied Mechanics and Engineering, 194:31473166, 2005.

[KK01] R. Kornhuber and R. Krause. Adaptive multigrid methods for Signorini's problem in linear elasticity. Comp. Vis. Sci., 4(1):9-20, 2001.

[KO88] N. Kikuchi and J.T. Oden. Contact problems in elasticity: a study of variational inequalities and finite element methods, volume 8 of SIAM Studies in Applied Mathematics. Society for Industrial and Applied Mathematics (SIAM), Philadelphia, PA, 1988.

[KS05] K. Kunisch and G. Stadler. Generalized Newton methods for the 2D-Signorini contact problem with friction in function space. M2AN Math. Model. Numer. Anal., 39(4):827$854,2005$.

[Kuč06a] R. Kučera. Minimizing quadratic functions with separable quadratic constraints. Optimization Methods and Software, to appear, 2006.

[Kuč06b] R. Kučera. A new algorithm for solving 3D contact problems with Coulomb friction. In Proceedings of the 16th International Conference on Domain Decomposition Methods 2005, New York, 2006.

[Lau02] T. Laursen. Computational Contact and Impact Mechanics. Springer Berlin Heildelberg, 2002.

[LPR91] C. Licht, E. Pratt, and M. Raous. Remarks on a numerical method for unilateral contact including friction. In Unilateral problems in structural analysis, IV (Capri, 1989), volume 101 of Internat. Ser. Numer. Math., pages 129-144. Birkhäuser, Basel, 1991.

[NJH80] J. Nečas, J. Jarušek, and J. Haslinger. On the solution of the variational inequality to the Signorini problem with small friction. Boll. Unione Math. Ital., 5:796-811, 1980.

[PL04] M. Puso and T. Laursen. A mortar segment-to-segment frictional contact method for large deformations. Comput. Methods Appl. Mech. Engrg., 193:4891-4913, 2004.

[QS93] L. Qi and J. Sun. A nonsmooth version of Newton's method. Math. Programming, 58(3, Ser. A):353-367, 1993.

[SGF00] O. Schenk, K. Gärtner, and W. Fichtner. Efficient sparse LU factorization with leftright looking strategy on shared memory multiprocessors. BIT, 40(1):158-176, 2000.

[Sta04] G. Stadler. Semismooth Newton and augmented Lagrangian methods for a simplified friction problem. SIAM J. Optim., 15(1):39-62, 2004.

[Wil03] K. Willner. Kontinuums- und Kontaktmechanik. Springer, 2003.

[WK03] B. Wohlmuth and R. Krause. Monotone methods on non-matching grids for non linear contact problems. SIAM Journal on Scientific Computing, 25:324-347, 2003.

[Wri02] P. Wriggers. Computational Contact Mechanics. John Wiley \& Sons, 2002.

S. HüEBER

Institut FÜr Angewandte Analysis und Numerische Simulation (IANS), Universität Stuttgart, Pfaffenwaldring 57, D-70569 Stuttgart, Germany

E-mail address: hueeber@ians.uni-stuttgart.de

G. StadleR

Center of Mathematics, University of Coimbra, Apartado 3008, 3001-454 Coimbra, Por-

TUGAL

E-mail address: georgst@mat.uc.pt 
B.I. Wohlmuth

Institut Für Angewandte Analysis und Numerische Simulation (IANS), Universität Stuttgart, Pfaffenwaldring 57, D-70569 Stuttgart, Germany

E-mail address: wohlmuth@ians.uni-stuttgart.de 